\title{
dmPFC-vIPAG projection neurons contribute to pain threshold maintenance and antianxiety behaviors
}

\author{
Jun-Bin Yin, ${ }^{1,2,3,4}$ Shao-Hua Liang, ${ }^{1,5}$ Fei Li, ${ }^{1,6}$ Wen-Jun Zhao, ${ }^{1,6}$ Yang Bai, ${ }^{1,3}$ Yi Sun, ${ }^{1,3,5}$ Zhen-Yu Wu, ${ }^{1,3}$ Tan Ding, ${ }^{7}$ Yan Sun, ${ }^{6}$ \\ Hai-Xia Liu, ${ }^{1}$ Ya-Cheng Lu, ${ }^{1}$ Ting Zhang, ${ }^{1}$ Jing Huang, ${ }^{1}$ Tao Chen, ${ }^{1}$ Hui Li,,${ }^{1,3}$ Zhou-Feng Chen, ${ }^{3}$ Jing Cao, ${ }^{8}$ Rui Ren, ${ }^{4}$ Ya-Nan Peng, ${ }^{4}$ \\ Juan Yang, ${ }^{4}$ Wei-Dong Zang, ${ }^{8}$ Xiang Li, ${ }^{9}$ Yu-Lin Dong, ${ }^{1}$ and Yun-Qing Li ${ }^{14,8}$ \\ 'Department of Anatomy, Histology and Embryology and K.K. Leung Brain Research Centre, The Fourth Military Medical University, Xi'an, China. 2Department of Neurology, the 960 ${ }^{\text {th }}$ Hospital of PLA, Jinan, \\ China. ${ }^{3}$ Center for the Study of Itch, Washington University School of Medicine, St. Louis, Missouri, USA. ${ }^{4}$ Key Laboratory of Brain Science Research and Transformation in the Tropical Environment of Hainan \\ Province, Haikou, China. 'Department of Human Anatomy, Binzhou Medical College, Yantai, China. ${ }^{6}$ Cadet Brigade, and 'Department of Orthopedics, Xijing Hospital, the Fourth Military Medical University, Xi'an, \\ China. ${ }^{8}$ Department of Anatomy, Basic Medical College, Zhengzhou University, Zhengzhou, China. ${ }^{9}$ Department of Orthopaedics, The First Affiliated Hospital of Nanjing Medical University, Nanjing, China.
}

\begin{abstract}
The dorsal medial prefrontal cortex (dmPFC) has been recognized as a key cortical area for nociceptive modulation. However, the underlying neural pathway and the function of specific cell types remain largely unclear. Here, we show that lesions in the dmPFC induced an algesic and anxious state. Using multiple tracing methods including a rabies-based transsynaptic tracing method, we outlined an excitatory descending neural pathway from the dmPFC to the ventrolateral periaqueductal gray (vIPAG). Specific activation of the dmPFC/vIPAG neural pathway by optogenetic manipulation produced analgesic and antianxiety effects in a mouse model of chronic pain. Inhibitory neurons in the dmPFC were specifically activated using a chemogenetic approach, which logically produced an algesic and anxious state under both normal and chronic pain conditions. Antagonists of the $\mathrm{CABA}_{A}$ receptor $\left(C A B A_{A} \mathrm{R}\right)$ or mGluR1 were applied to the dmPFC, which produced analgesic and antianxiety effects. In summary, the results of our study suggest that the dmPFC/vIPAG neural pathway might participate in the maintenance of pain thresholds and antianxiety behaviors under normal conditions, while silencing or suppressing the dmPFC/vIPAC pathway might be involved in the initial stages and maintenance of chronic pain and the emergence of anxiety-like behaviors.
\end{abstract}

\section{Introduction}

Patients with chronic pain usually experience not only severe pain perception but also negative emotions and decreased cognitive abilities $(1,2)$. Intractable and permanent pain conditions easily develop into a "chronic pain cycle," and chronic pain induces negative emotions including anxiety and depression, sleep disorder, and reduced activity and also affects cognitive abilities (2-4). Conversely, negative emotions and reduced cognitive abilities further worsen chronic pain $(5,6)$. This chronic pain cycle has made it very difficult to treat chronic pain clinically. There might be a special neural circuit that mediates this vicious cycle, which would be a good target for the treatment of chronic pain. Many studies have focused on the emotional components of pain in an attempt to identify the neural pathway related to emotional pain (7-10). However, the underlying mechanisms for this chronic pain cycle are still unclear.

One useful way to address this chronic pain cycle would be to identify a special region or area in the cerebral cortex that is

Authorship note: JBY, SHL, and FL contributed equally to this work. Conflict of interest: The authors have declared that no conflict of interest exists. Copyright: () 2020, American Society for Clinical Investigation.

Submitted: February 2, 2019; Accepted: August 20, 2020; Published: November 3, 2020. Reference information: J Clin Invest. 2020;130(12):6555-6570.

https://doi.org/10.1172/JCl127607. involved in the modulation of pain, emotions, and cognition. The dorsal medial prefrontal cortex (dmPFC), including the rostral anterior cingulate cortex (rACC) and prelimbic cortex (PL), is a region or area involved in all of these processes (11-13). Many previous studies have demonstrated that the functional deactivation of the dmPFC induces negative emotions and decreases cognitive abilities $(14,15)$. A few studies have also suggested that deactivation of the dmPFC enhances pain perception $(8,10,16)$. Functional and morphological abnormalities in the dmPFC have been documented in an animal model of chronic pain $(17,18)$. Both background and evoked activities of $\mathrm{dmPFC}$ neurons were found to be decreased in an arthritis-induced chronic pain model (19, 20). It has been speculated that the progression of pain from an acute to a chronic state is accompanied by a decrease in $\mathrm{dmPFC}$ activity $(21,22)$. This pain-related inhibition of $\mathrm{dmPFC}$ neurons in the arthritic model depended on metabotropic glutamate receptor 1-mediated (mGluR1-mediated) endogenous activation of $\gamma$-aminobutyric acid receptor subtype A $\left(G_{A B A} R\right)(19)$. However, the exact neural pathway from the dmPFC that is involved in pain modulation has only begun to be revealed (23). The periaqueductal gray/rostral ventromedial medulla/spinal dorsal horn (PAG/RVM/SDH) pathway is one of the most important neural pathways involved in descending pain modulation (24-26). It has been demonstrated that specific activation of the vesicular glutamate transporter 2-immunoreactive (VGLUT2-ir) neurons in the 
A

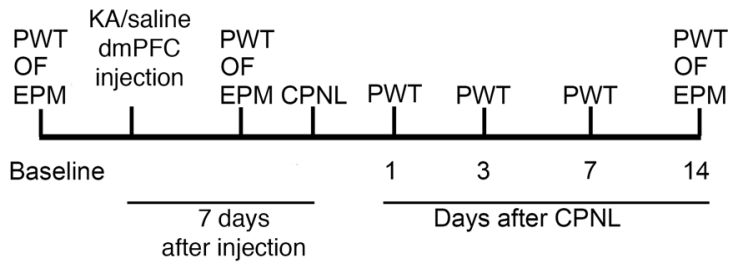

B

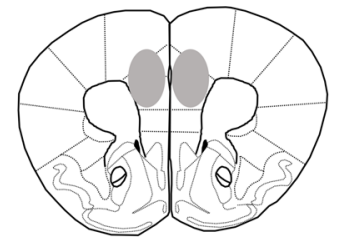

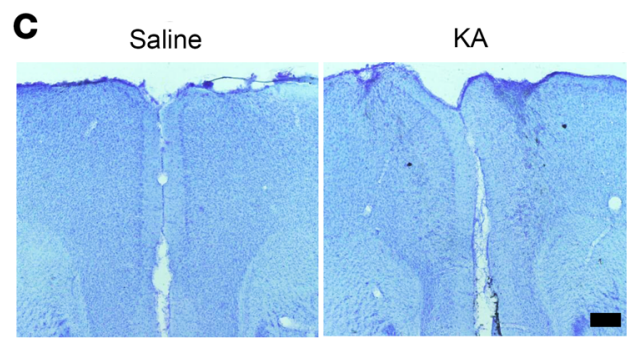

$\mathbf{F}$
D
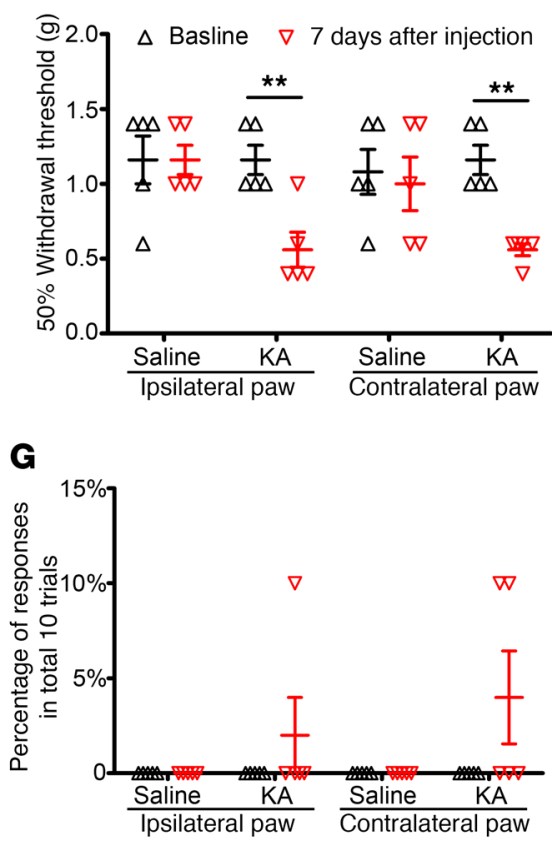

J

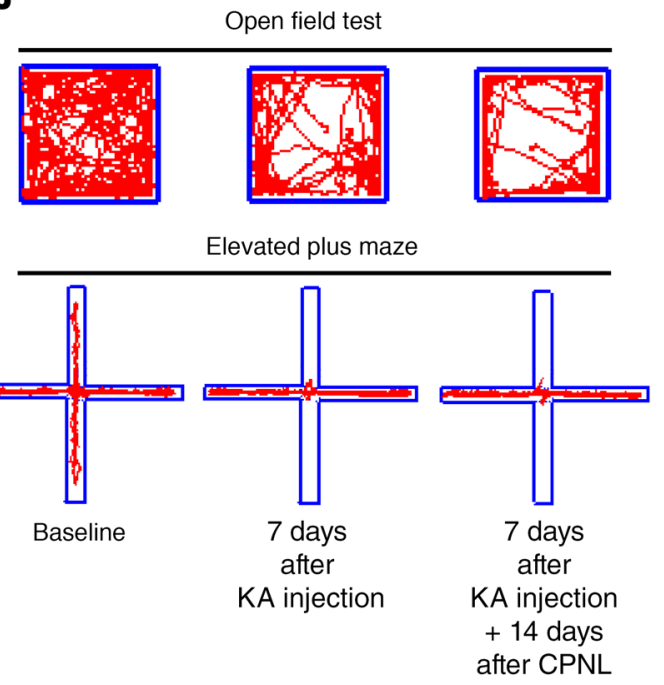

E

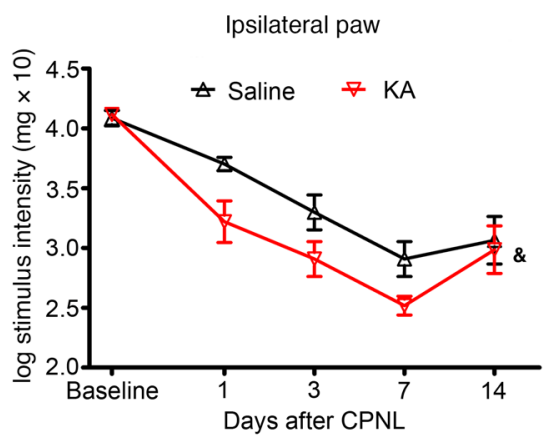

H

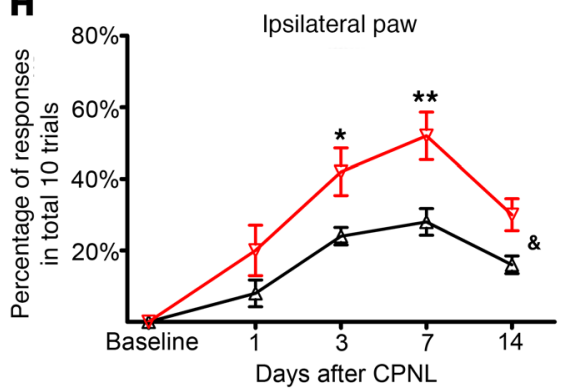

K

$\Delta$ Baseline $\quad \nabla 7$ days after injection
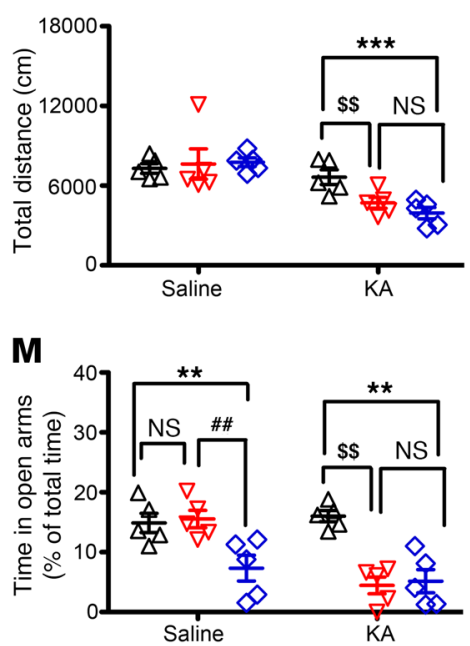

Contralateral paw
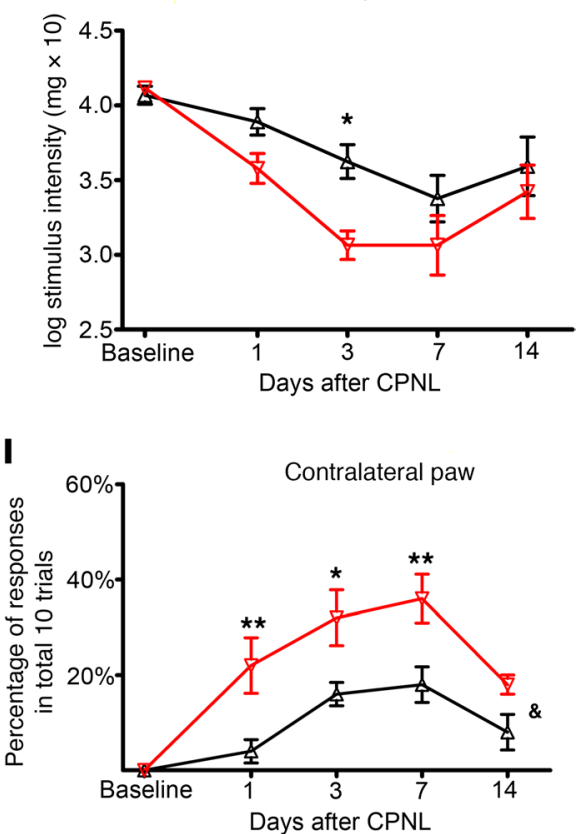

$\mathbf{L}$
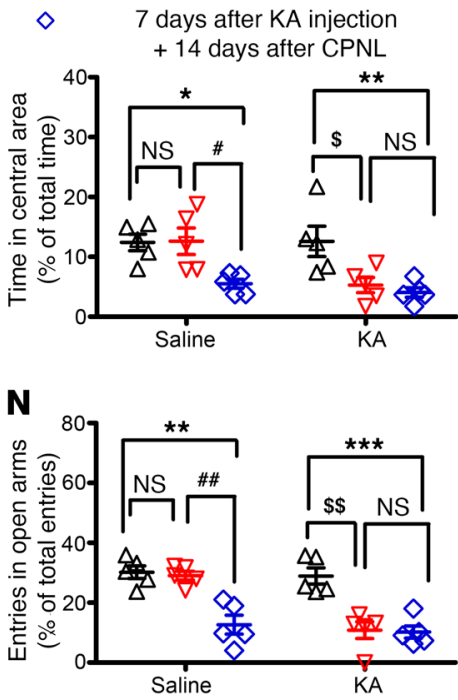
Figure 1. Bilateral lesions in the dmPFC worsen CPNL-induced mechanical nociception and anxiety-like behaviors. (A and B) Representative schematic diagrams of the KA injection into the dmPFC and procedures for the behavioral tests. PWT, paw withdrawal threshold. (C) Nissl staining of the dmPFC after KA or saline dmPFC injections. Scale bar: $200 \mu \mathrm{m}$. (D) Mechanical thresholds at baseline and 7 days after saline or KA dmPFC injections. ${ }^{*} P<0.01$, by paired $t$ test $(n=5)$. Mechanical thresholds of the ipsilateral $(\mathbf{E})$ and contralateral $(\mathbf{F})$ hind paws following $C P N L$ with saline or KA dmPFC injections (E: $F_{(1,32)}=6.07, P=0.04 ;{ }^{\&} P<0.05 ; \mathbf{F}: F_{(1,32)}=4.22, P=$ 0.07 ; ${ }^{*} P<0.05$; 2-way, repeated-measures ANOVA with Bonferroni's post hoc test $(n=5)$. (G) Responses to non-nociceptive stimulation at baseline and 7 days after saline or KA dmPFC injections $(n=5)$. Responses to non-nociceptive stimulation of the ipsilateral $(\mathbf{H})$ and contralateral $(\mathbf{I})$ hind paws following CPNL with saline or KA dmPFC injections $\left(\mathbf{H}: F_{(1,32)}=9.80\right.$, $P=0.014 ;{ }^{\&} P<0.05 ;$ I: $F_{(1,32)}=10.68, P=0.011 ; \& P<0.05 ; 2$-way, repeated-measures ANOVA with Bonferroni's post hoc test, ${ }^{*} P<0.05$,

${ }^{* *} P<0.01(n=5)$. (J) Schematic traces of the OF and EPM tests at baseline, 7 days after KA injection, and 7 days after KA injection plus 14 days after CPNL. Total distance traveled (K) and percentage of time spent in the central area (L) in the OF test (saline injection, $\mathbf{K}: F_{(2,8)}=0.11, P=0.90$; L: $F_{(2,8)}=5.97, P=0.03 ; \mathrm{KA}$ injection, K: $F_{(2,8)}=24.97, P<0.001 ; \mathrm{L}: F_{(2,8)}=9.33$, $P<0.01$; 1-way, repeated-measures ANOVA with Tukey's post hoc test, ${ }^{*} P$ $<0.05$, ${ }^{*} P<0.01$, and ${ }^{* * *} P<0.001 ;{ }^{\$} P<0.05$ and ${ }^{\$} P<0.01$; ${ }^{*} P<0.05$ ( $n$ $=5)$. Percentage of time in $(\mathbf{M})$ and percentage of entries into $(\mathbf{N})$ the $O A s$ in the EPM test (saline injection, $\mathbf{M}: F_{(2,8)}=13.22, P<0.01 ; \mathbf{N}: F_{(2,8)}=20.28$, $P<0.001$; KA injection, $\mathbf{M}: F_{(2,8)}=17.34, P<0.01 ; \mathbf{N}: F_{(2,8)}=23.23, P<0.001$; 1-way, repeated measures ANOVA with Tukey's post hoc test, ${ }^{* *} P<0.01$ and ${ }^{* *} P<0.001 ;{ }^{\$ \$} P<0.01 ;{ }^{\# \#} P<0.01(n=5)$.

ventrolateral subregion of the PAG (vlPAG), the region of the PAG that has been most closely linked to descending pain modulation, produces clear analgesic effects, which indicates that VGLUT2-ir neurons in the vlPAG are key relay points for descending pain modulation $(27,28)$. We hypothesized that dmPFC neurons project to the vlPAG and participate in descending pain modulation, given the abundant projections from the superior nucleus in the frontal cortex to the vlPAG. Thus, the deactivation of the $\mathrm{dmPFC} / \mathrm{vlPAG}$ neural pathway would induce a chronic pain cycle and lead to increased suffering of patients with chronic pain.

To test whether this hypothesis is correct, we applied chemical lesion, optogenetic, chemogenetic, molecular biological, and behavioral pharmacological approaches to demonstrate the involvement of the descending dmPFC/vlPAG pathway in pain modulation and negative emotion processing. In the present study, as a first step, we used many types of morphological approaches to establish the dmPFC/vlPAG neural pathway and investigate the chemical properties of the projecting neurons. We also carried out functional investigations to determine whether the dmPFC/ vlPAG neural pathway is involved in pain-related and anxiety-like behaviors. Finally, different methods were used within the $\mathrm{dmPFC}$ to reveal the underlying molecular mechanisms related to this descending pain modulation.

\section{Results}

Bilateral dmPFC lesions promote nociception under normal and chronic pain conditions. To determine whether the dmPFC participates in pain perception, we injected kainic acid (KA) into the bilateral dmPFC of mice to induce cell damage. After KA injection, we evaluated the mechanical thresholds of the mice under normal and chronic pain conditions (Figure 1, A and B). We observed reduced densities and disordered arrangements of cells in the dmPFC following KA injection (Figure 1C). Loss of neuronal cell bodies and proliferation of astrocytes were also indicators of dmPFC lesions (Supplemental Figure 1; supplemental material available online with this article; https://doi.org/10.1172/JCI127607DS1). Under normal conditions, the dmPFC lesion significantly reduced the mechanical thresholds of both hind paws (Figure 1D), and the mice showed obvious mechanical hyperalgesia. After the common peroneal nerve ligation (CPNL) model was established, bilateral dmPFC lesions significantly facilitated CPNL-induced hyperalgesia (Figure 1, E and F). However, the dmPFC lesion had no influence on the responses to non-noxious mechanical stimulation (Figure 1G), which indicates that the $\mathrm{dmPFC}$ lesions in naive mice did not induce allodynia. The responses of both hind paws to non-noxious stimuli in mice in the lesion group were significantly higher than those in mice in the saline group (Figure 1, H and I).

Bilateral dmPFC lesions induce anxiety-like behaviors. Chronic pain is often accompanied by anxiety-like behaviors. Therefore, we assessed the effects of the CPNL-induced dmPFC lesion on the animals' anxiety-like behaviors. Mice subjected to CPNL spent a decreased percentage of time in the central area of the open field (OF) 14 days after CPNL; in contrast, the total distance traveled in the OF was unaffected (Figure 1, J-L). However, the dmPFC lesion significantly decreased the total distance traveled in the OF test (Figure 1, J and K). Interestingly, we found that CPNL did not induce more anxiety-like behaviors in the OF test after induction of the dmPFC lesion (Figure $1, \mathrm{~K}$ and $\mathrm{L}$ ). Then, we further tested the effects of the dmPFC lesion on CPNL-induced anxiety-like behaviors in the elevated plus maze (EPM) test. CPNL decreased both the percentage of time spent in the open arms (OAs) and the percentage of entries into the OAs in the EPM (Figure 1, J, M, and N). Similarly, after the dmPFC lesions, mice subjected to CPNL did not show more anxiety-like behaviors in the EPM test (Figure 1, M and N). These results demonstrate that CPNL-induced anxiety-like behaviors did not occur after neurons were damaged in the dmPFC. Although we have not investigated the underlying mechanism, a possible explanation for this result was the floor effect.

Projections from the dmPFC terminate onto VGLUT2-ir neurons within the vlPAG. Given the important roles of the dmPFC in the modulation of nociception and anxiety-like behaviors, it was critical to investigate the direct connections between the dmPFC and the vlPAG, which is known to play an important role in pain modulation. Fluoro-Gold (FG) was injected into the vlPAG. We observed many FG-labeled projection neurons in the dmPFC, which also exhibited CaMKII immunoreactivity and expressed VGLUT1 mRNA (Supplemental Figures 2 and 3). Moreover, the FG-labeled neurons did not express both VGLUT2 mRNA and GAD67. Double staining of NeuN and FG showed that the projection neurons were mainly distributed in layers $\mathrm{V}$ and $\mathrm{VI}$ in the dmPFC (Figure 2, A-D). These results indicated that there were direct innervations from the dmPFC excitatory pyramidal neurons to the vlPAG. To identify the synaptic connections, we applied a rabies-based, retrograde, transsynaptic tracing approach to identify the dmPFC monosynaptic inputs onto VGLUT2-ergic neurons in the vlPAG, using a transgenic mouse line expressing Cre recombinase in these neurons (Figure 2, E-H). The starter neurons (GFP 
A

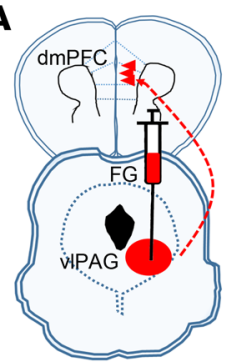

$\mathbf{F}$

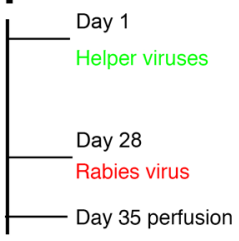

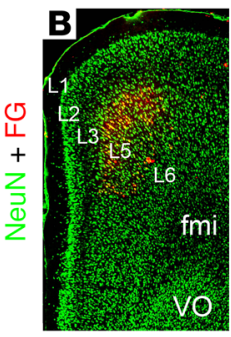

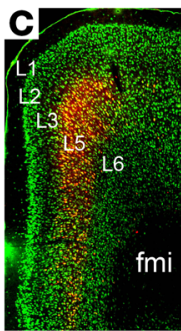

G

Helper viruses: AAV-DIO-GFP-TVA

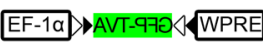
AAV-DIO-RVG EF-1a एVЯ WPRE

Rabies virus: RV SADAG-dsRed (EnvA)

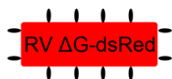

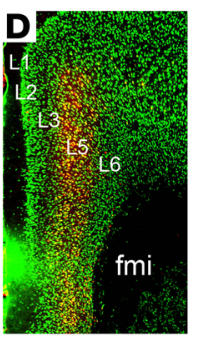

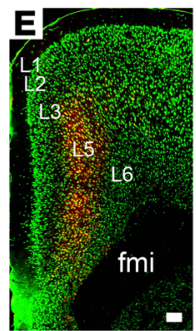

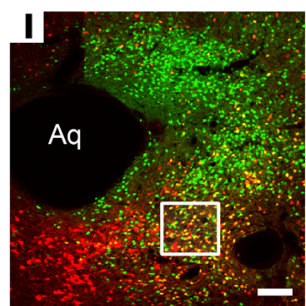
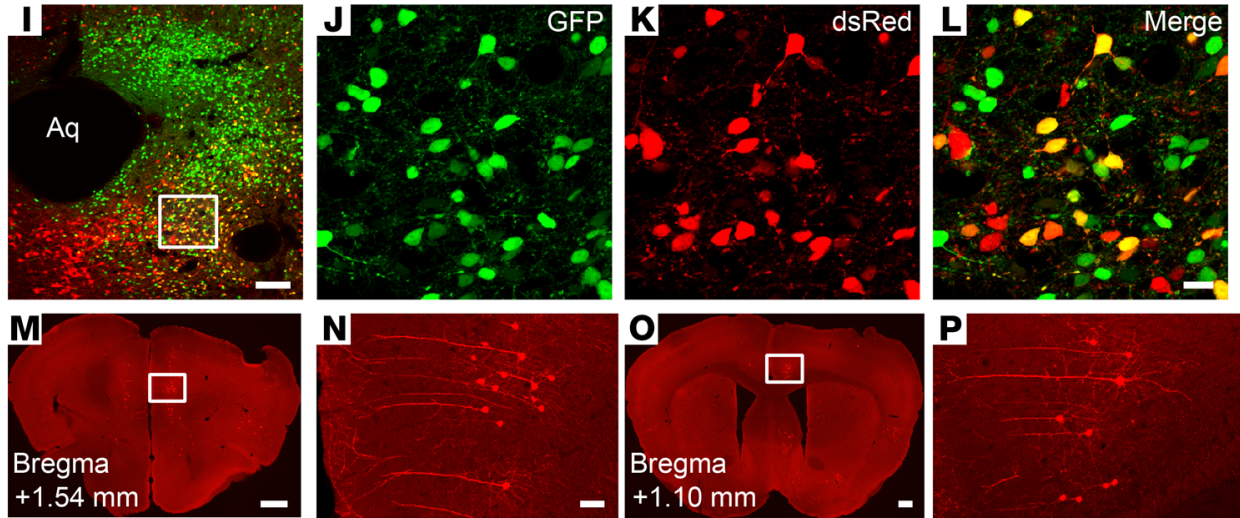

Figure 2. Projections from the dmPFC to the VGLUT2-containing neurons in the vIPAG. (A) Schematic diagram of retrograde tracing of projection neurons in the dmPFC, which were labeled with FG injected into the vIPAG. (B-E) Images show different coronal dmPFC sections with double staining of FG (red) injected into the vIPAG and NeuN (green). L1-L6, layers 1-6; fmi, forceps minor of the corpus callosum; V0, ventral orbital cortex. Scale bar: 100 $\mu$ m. (F-H) Schematics of rabies-based transsynaptic tracing. (F and G) VGLUT2-Cre mice were transduced with 2 AAVs in the vIPAG followed by EnvA-pseudotyped, rabies virus glycoprotein-deleted (RVG-deleted), and dsRed-expressing rabies virus. (H) Schematic of sagittal section of the mouse brain shows VGLUT2 starter cells labeled in yellow and presynaptic partners throughout the brain in red. (I-L) Coronal sections through the vIPAC of a brain with VGLUT2-Cre tracing show the location of starter cells (yellow), which were double stained with GFP (green) and dsRed (red). Scale bars: $100 \mu \mathrm{m}$ (I); $20 \mu \mathrm{m}$ (enlarged insets in J-L). Aq, aqueduct. (M-P) Images of a coronal section through the dmPFC and a section through the ACC show dsRed-labeled presynaptic projection neurons. The areas in the white frames in $\mathbf{M}$ and $\mathbf{O}$ were magnified in $\mathbf{N}$ and $\mathbf{P}$. Scale bars: $300 \mu \mathrm{m}$ ( $\mathbf{M}$ and $\mathbf{0}) ; 50 \mu \mathrm{m}$ ( $\mathbf{N}$ and $\mathbf{P}$ ). All experiments were repeated 3 times and yielded consistent results.

and dsRed double-stained neurons) were localized in the vlPAG (Figure 2, I-L). In the sections of the forebrain, we observed many dsRed transsynaptic-labeled pyramidal projection neurons in the $\mathrm{dmPFC}$ and anterior cingulate cortex (ACC) (Figure 2, M-P). The above retrograde tracing results suggested that the excitatory pyramidal neurons in the dmPFC project to the VGLUT2-ir neurons in the vlPAG.

As with the retrograde tracing experiments (Figure 3A), we injected the anterograde tracer biotinylated dextran amine (BDA) into the $\mathrm{dmPFC}$ and observed dense BDA-labeled fibers and terminals in the vlPAG (Supplemental Figure 4). For further confirmation, we injected adeno-associated virus 2/2-CaMKII $\alpha-$ enhanced yellow fluorescent protein (AAV2/2-CaMKII $\alpha$-EYFP) into the dmPFC to infect the excitatory pyramidal neurons (Figure 3, B and C). After 3 weeks, FG was injected into the RVM. We observed many EYFP-labeled fibers and terminals in the vlPAG (Figure 3D). FISH identified the FG-labeled RVM projection neurons in the vlPAG, and most of them expressed VGLUT2 mRNA (Figure 3, E-H). Moreover, the FG-labeled SDH projection neurons in the RVM expressing 5-HT received many VGLUT2-ir contacts (Supplemental Figures 5 and 6). To confirm the links between dmPFC and the PAG-RVM descending pain modulation system, AAV2/2-CaMKII $\alpha$-EYFP was injected into the dmPFC, and FG was injected into the RVM. FG-labeled RVM projection neurons in the vlPAG received the EYFP-labeled fiber contacts, which colocalized with the presynaptic marker synapsin (Figure 3, I-L). We also applied an anterograde transsynaptic tracing approach, following that used in previous studies $(29,30)$, to identify the dmPFC monosynaptic inputs onto FG-labeled RVM projection neurons in the vlPAG (Figure 3, M-P, and Supplemental Table 1). 
A
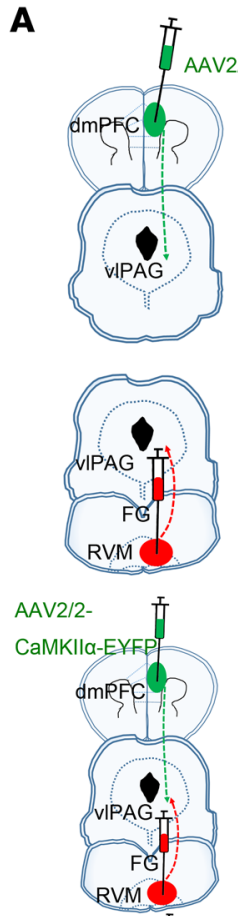

SCAAV2/1-hSyn- I FLEX-EGEP

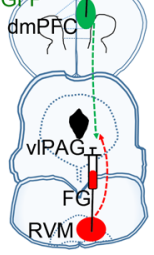

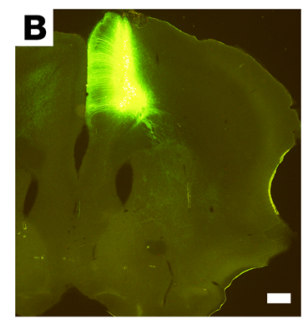
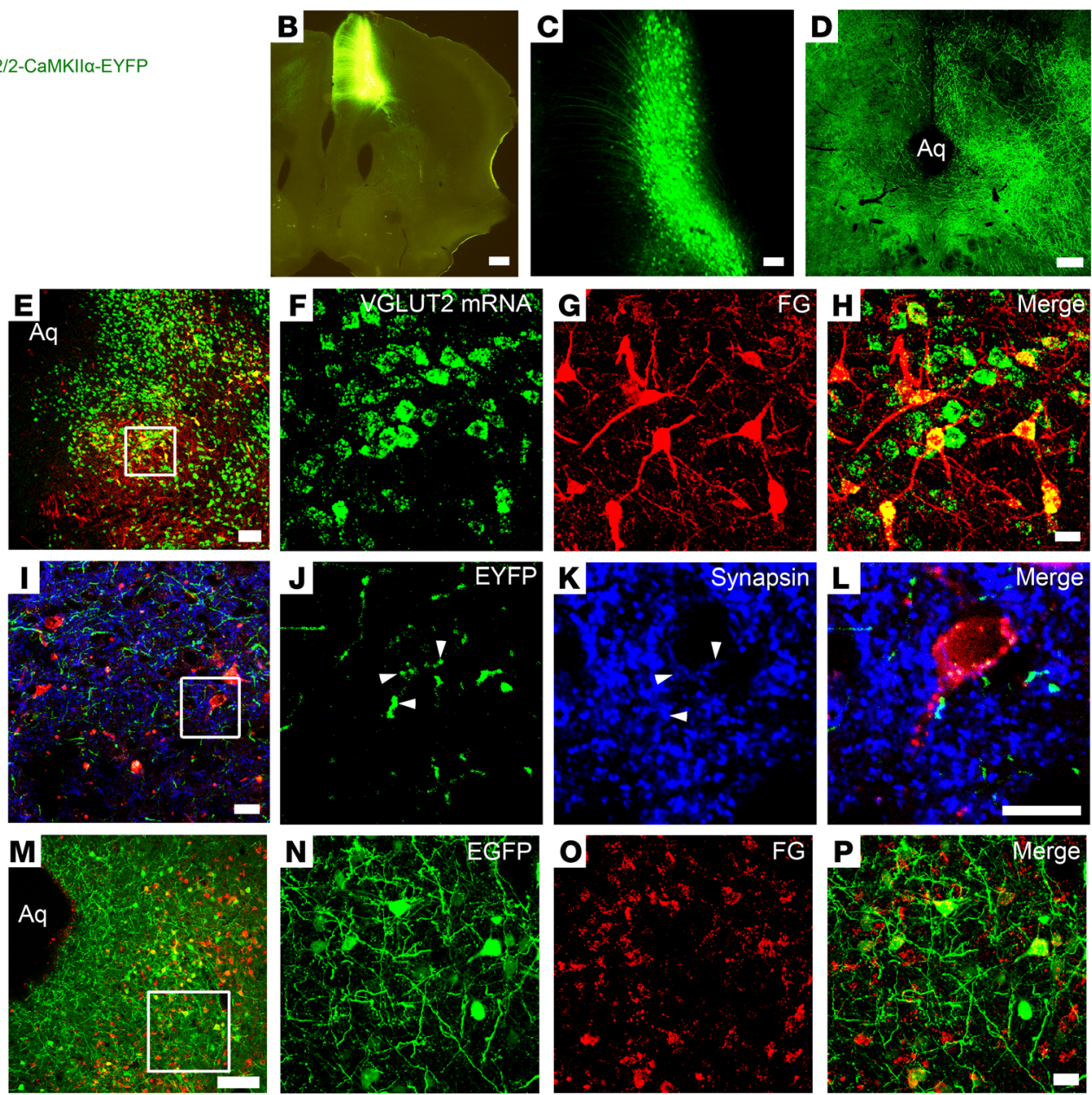

Figure 3. Light microscope observation of the dmPFC/vIPAC/RVM neural pathway. (A) Schematic diagram of the dmPFC/vIPAC/RVM neural pathway demonstrating use of various types of tracers and viruses. (B and C) Infection images of AAV2/2-CaMKIl $\alpha$-EYFP in the dmPFC. (D) The distributions of EYFP-labeled fibers coming from the dmPFC pyramidal neurons in the vIPAG. Scale bars: $100 \mu \mathrm{m}$ (B); $50 \mu \mathrm{m}$ (C); $200 \mu \mathrm{m}$ (D). (E-H) RVM projection neurons labeled with hybridization signals for VGLUT2 mRNA in the vIPAG. The hybridization signals were visualized with FITC (green), whereas FG-ir was visualized with Alexa Fluor 594 (red). Scale bars: $100 \mu \mathrm{m}$ (E); $10 \mu \mathrm{m}$ (F-H) . (I-L) Triple-staining of EYFP-labeled fibers (green), FG-ir projection neurons (red), and synapsin-ir terminals (blue) in the vIPAG showing the relationship between fibers projecting from the dmPFC and neurons projecting to the RVM. White arrowheads indicate EYFP-labeled fibers that also expressed synapsin and contacted FG-ir projection neurons. Scale bars: $100 \mu \mathrm{m}$ (I); $20 \mu \mathrm{m}$ (J-L). (M-P) Anterograde transsynaptic virus EGFP-labeled neurons (green) also expressed FG-ir (red), which indicated that the vIPAG-RVM projection neurons received direct innervation by the dmPFC. Scale bars: $100 \mu \mathrm{m}(\mathbf{M}) ; 20 \mu \mathrm{m}(\mathbf{N}-\mathbf{P})$. All experiments were repeated 3 times and yielded consistent results.

Again, these tracing studies indicated that there exists a descending pain modulation pathway: the $\mathrm{dmPFC/vlPAG/RVM} \mathrm{pathway.}$

Hypoactivity in dmPFC-vlPAG projection neurons of CPNLtreated mice. To examine how CPNL affects the neuronal activity of dmPFC-vlPAG projection neurons, we performed whole-cell patch-clamp recordings from dmPFC-vlPAG projection neurons of control and CPNL-treated mice (Figure 4, A-F). Excitability of dmPFC-vlPAG projection neurons was decreased in the CPNL group (Figure 4, $\mathrm{G}$ and $\mathrm{H}$ ). Importantly, the $\mathrm{GABA}_{\mathrm{A}} \mathrm{R}$ blocker bicuculline $(20 \mu \mathrm{M})$ reversed the low activity in dmPFC-vlPAG projection neurons, indicating that the observed CPNL-induced reduction in dmPFC-vlPAG projection neurons activity was mediated by enhanced GABAergic inputs (Figure 4, G and H). Altogether, these data indicate that CPNL triggered long-term $G_{A B A} R$ mediated inhibition in the dmPFC-vlPAG projection neurons.

Analgesic and antianxiety effects of specific activation of the dmPFC/vlPAG neural pathway. Given the CPNL-induced hypo- activity we observed in the dmPFC/vlPAG pathway, we became very interested in the role of the $\mathrm{dmPFC} / \mathrm{vlPAG}$ neural pathway in pain modulation and pain-related anxiety-like behaviors. Following specific activation of the dmPFC/vlAPG pathway using an optogenetic approach, the mechanical thresholds of the bilateral hind paws were tested 7 days after CPNL, and behaviors in the OF and EPM tests were assessed 14 days after CPNL (Figure 5, A and B). After light stimulation, we observed a greater number of neurons expressing FOS-ir around the EYFP fibers in the vlPAG (Figure 5, C-F). This indicates that the neurons in the vlPAG were activated by stimulation of the fibers coming from the dmPFC. We noted obvious analgesic effects not only on the ipsilateral hind paw but also on the contralateral hind paw in the CPNL-treated mice, following specific activation of the $\mathrm{dmPFC} / \mathrm{vlPAG}$ pathway (Figure 5G). However, in the CPNL-treated mice infected with AAV2/2-CaMKII $\alpha$-EYFP, the light stimulation had no effects on the mechanical thresholds. Unexpectedly, activation of the dmPFC/ 
A

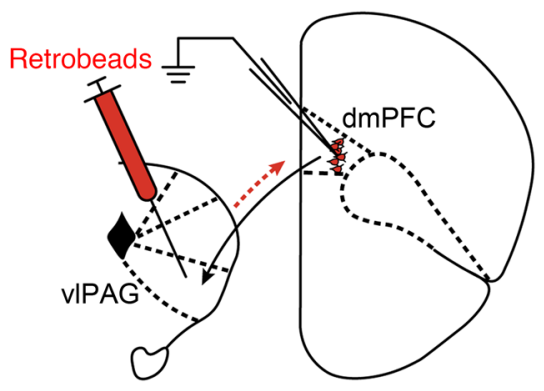

G
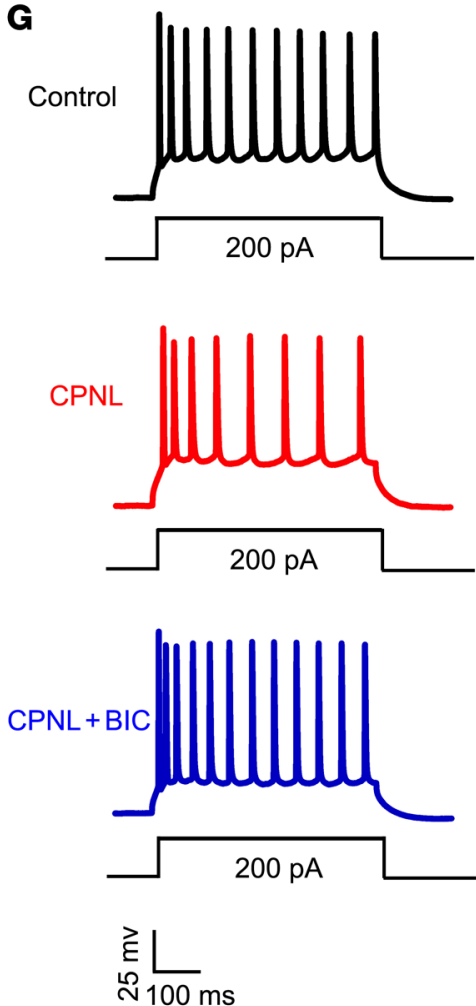
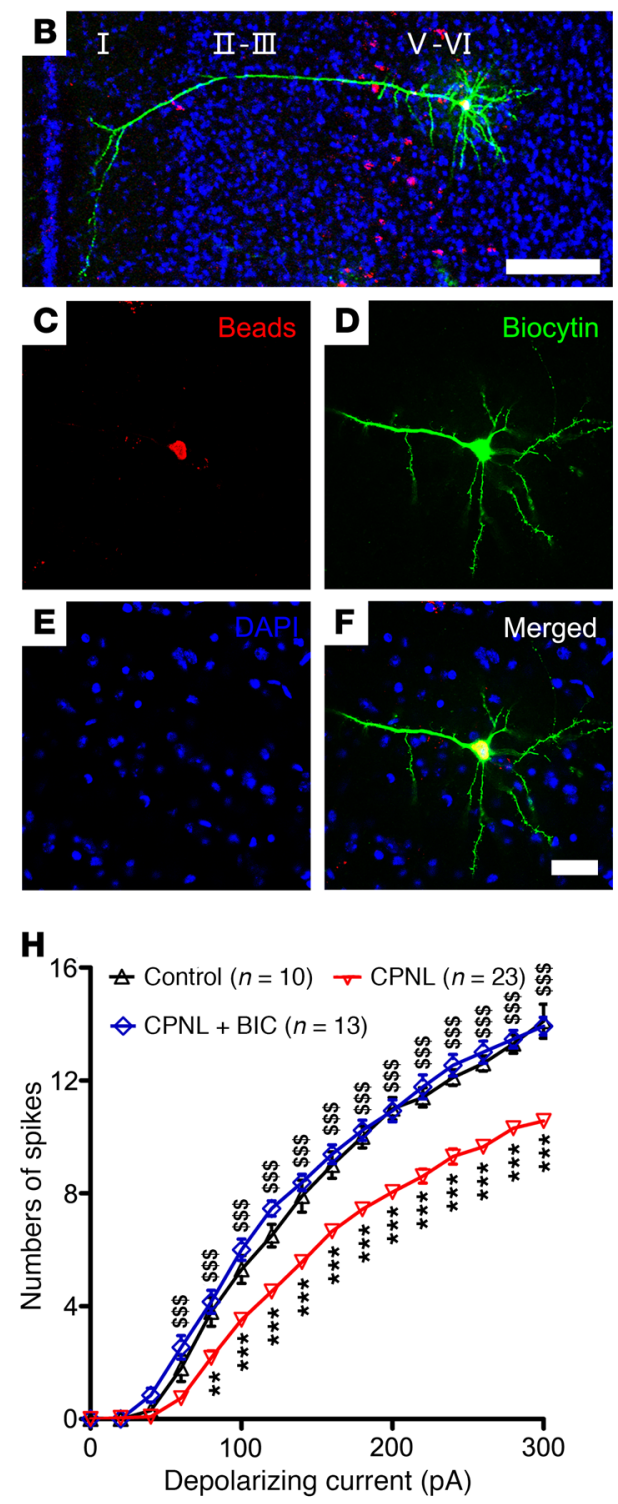

Figure 4. Neuronal activity recorded in dmPFC-vIPAG projection neurons in CPNL- and sham-treated mice. (A) Schematic diagram of electrophysiological recording of neurons in the dmPFC, which were labeled with Retrobeads injected into the vIPAC. (B-F) Triple staining of biocytin-filled neurons (green, indicating recorded neurons), bead-labeled neurons (red, indicating dmPFC-vIPAC projection neurons), and DAPI (blue) in the dmPFC after electrophysiological recording. Scale bars: $200 \mu \mathrm{m}$ (B); $50 \mu \mathrm{m}$ (C-F). (C) Representative recordings of action potential firing of dmPFC-vIPAC projection neurons in response to current injection (200 pA) in control, CPNL, and CPNL plus bicuculline (BIC) groups. (H) Averaged action potentials of dmPFC-vIPAC projection neurons, induced by step-current injections (0-300 pA). Note the decrease in excitability of dmPFC-vIPAC projection neurons in the CPNL mice and its reversal by bicuculline $(20 \mu \mathrm{M})$. Control group versus the CPNL group, ${ }^{* *} P<0.005$ and ${ }^{* *} P<0.001$; CPNL group versus the CPNL plus bicuculline group, ${ }^{\$ \$} P<0.001 ; 2$-way, repeated-measures ANOVA with Bonferroni's post hoc test.

vlPAG pathway had no obvious effects on either the total distance traveled or the percentage of time spent in the central area in the OF test performed 14 days after CPNL (Figure 5, $\mathrm{H}$ and I). However, both the reduced percentage of time spent in (Figure 5J) and the percentage of entries into (Figure 5K) the OAs in the EPM test were significantly reversed during blue light stimulation for the mice that received the hChR2-EYFP virus injection. These optogenetic results suggest that specific activation of the $\mathrm{dmPFC} / \mathrm{vlPAG}$ neural pathway produced analgesic and antianxiety effects in the
CPNL model. The dmPFC/vlPAG neural pathway was involved in the modulation of nociception and anxiety-like behaviors.

Specific activation of inhibitory neurons in the dmPFC facilitates nociception and anxiety-like behaviors. Activation of the local inhibitory neurons potentially suppressed pyramidal neurons within the $\mathrm{dmPFC}$ and resulted in deactivaton of the dmPFC/-vlPAG pathway. We introduced the designerreceptorsexclusivelyactivated by designer drugs (DREADDs) method to investigate the functional roles of $\mathrm{dmPFC}$ local inhibitory neurons in modulating nociception and anxiety-like behaviors (Figure 6, A and B). The triple-labeling results showed that many GAD67-ir inhibitory neurons expressed mCitrin and FOS (Figure 6, C-J), which indicated that the inhibitory neurons were certainly activated after the clozapine- $N$-oxide (CNO) i.p. injection. Under normal conditions, specific activation of inhibitory neurons in the dmPFC clearly reduced the mechanical thresholds of the bilateral hind paws (Figure 6, K and L). After CPNL was established, CNO i.p. injection further reduced the mechanical thresholds of the bilateral hind paws in mice that received the hM3Dq-mCitrin injection (Figure 6, K and L). The above results suggest that activation of inhibitory neurons in the $\mathrm{dmPFC}$ produced hyperalgesic states under both normal and chronic pain conditions.

Then, we tested the effects of specific activation of local inhibitory neurons on anxiety-like behaviors in the OF and EPM under normal and chronic pain conditions. Under normal conditions, both the total distance traveled (Figure 6M) and the percentage of time in the central area (Figure $6 \mathrm{~N}$ ) in the OF test were significantly decreased by specifically activating inhibitory neurons in the dmPFC. In the EPM test, CNO injections also significantly suppressed the percentage of time in (Figure 6O) and the percentage of entries into (Figure 6P) the OAs for the mice that received hM3Dq-mCitrin virus injections. Fourteen days after CPNL, the total distance traveled in the OF test was significantly reduced following specific activation of inhibitory neurons (Figure 6Q), and the percentage of time in the central area in the OF test was also clearly further suppressed (Figure 6R). In the EPM test, the CNO injection also significantly further suppressed the percentage of time spent in (Figure 6S) and the percentage of entries into (Figure 6T) the OAs for the mice that received the hM3Dq-mCitrin virus injection. These observations indicate 
A

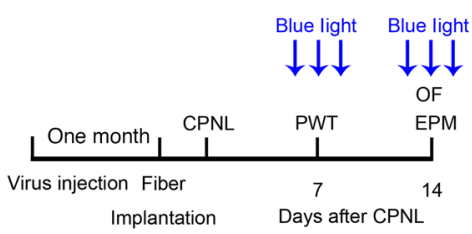

B
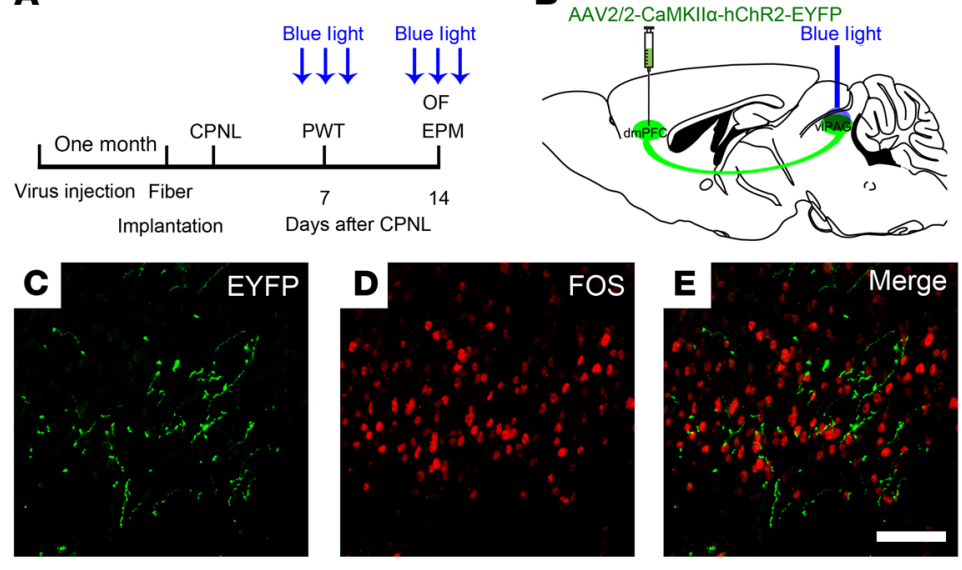

$\mathbf{F}$

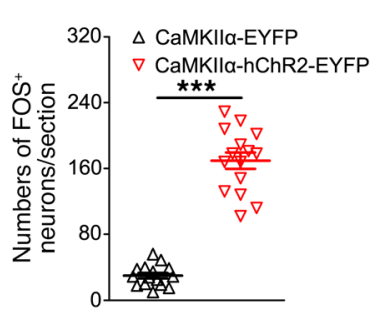

G

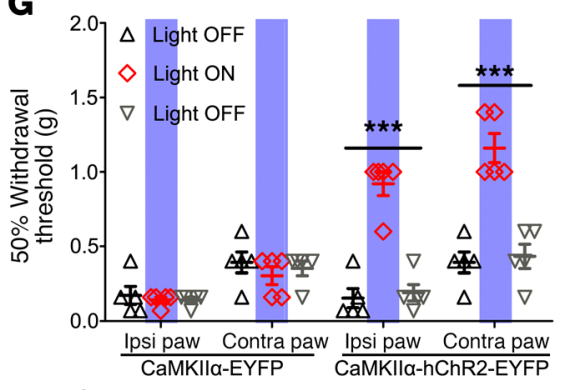

H
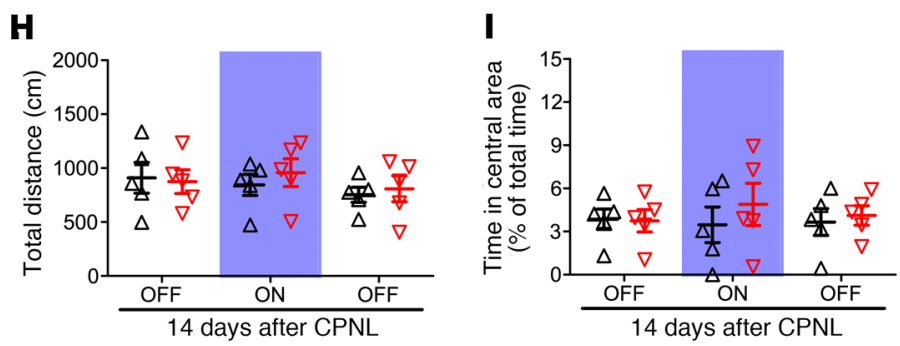

$\mathbf{J}$
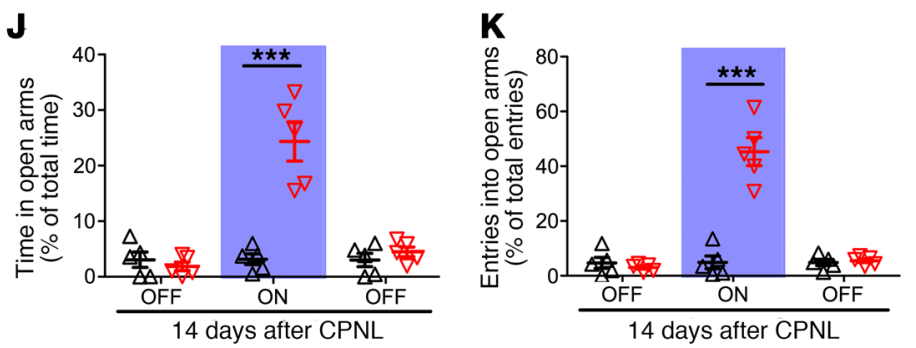

Figure 5. Optogenetic activation of the dmPFC/vIPAG neural pathway attenuates CPNL-induced mechanical hyperalgesia and anxiety-like behaviors. (A and B) Representative schematic diagrams of optogenetic activation of the dmPFC/vIPAC neural pathway and the procedures for behavioral testing. (C-E) Double staining of EYFP-labeled fibers (green) and FOS-ir neurons (red) in the vIPAC after blue light stimulation. Scale bar: $50 \mu \mathrm{m}$. (F) Mean number of FOS-ir neurons in the vIPAC following optogenetic activation ( ${ }^{* *} P<0.001$; Student's $t$ test; $n=5$ mice per group). (G) Mechanical thresholds of the bilateral hind paws for CPNL-treated mice after AAV2/2-CaMKIl $\alpha$-EYFP or AAV2/2-CaMKIl $\alpha$-hChR2EYFP virus injection, with $473 \mathrm{~nm}$ blue light off-on-off stimulation (ipsilateral [Ipsi], control virus: $F_{(2,8)}=0.29, P=0.75$; hChR2 virus: $F_{(2,8)}=57.57, P<0.001$; contralateral [Contra], control virus: $F_{(2,8)}=$ $0.50, P=0.62$; hChR2 virus: $F_{(2.8)}=42.79, P<0.001$; ${ }^{* *} P<0.001$, 1 -way, repeated-measures ANOVA with Tukey's post hoc test; $n=$ 5 mice per group). (H and I) Total distance traveled and percentage of time spent in the central area of the OF test with 3-minute light off, 3-minute light on, and 3-minute light off periods $\left(\mathbf{H}: F_{(1,16)}=\right.$ 1.09, $P=0.33, t=0.70, P>0.05 ; \mathrm{I}: F_{(1,16)}=0.40, P=0.54, t=0.99, P$ $>0.05$; 2-way, repeated-measures ANOVA, with Bonferroni's post hoc tests; $n=5$ mice per group). (J and K) Percentage of time spent in and percentage of entries into the OAs in the EPM test with 3-minute light off, 3-minute light on. and 3-minute light off periods (J: $F_{(1,16)}=21.12, P<0.01, t=8.65, P<0.001 ; \mathbf{K}: F_{(1,16)}=47.95, P<$ $0.001, t=11.37, P<0.001$; ${ }^{* *} P<0.001,2$-way, repeated-measures ANOVA with Bonferroni's post hoc tests; $n=5$ mice per group).

(red) in the dmPFC (Figure 7C). A separate group of animals was prepared for functional characterization of the hChR2 and hM3Dq viruses. Blue light activation of the hChR2 virus in acute brain slices reliably elicited action potential spikes in inhibitory neurons following different stimulation frequencies (Figure 7D). A bath application of $40 \mu \mathrm{M}$ CNO induced a transient depolarization and robust action potential firing in dmPFC-vlPAG projection neurons (Figure 7E). When we recorded the projection neurons, we found that light stimulation induced obvious inhibitory postsynaptic currents (IPSCs) (Figure $7 F)$. Picrotoxin (PTX), an antagonist of the $\mathrm{GABA}_{\mathrm{A}} \mathrm{R}$, completely blocked the light-evoked IPSCs (Figure 7G). Seven days after CPNL was established, we found that CNO i.p. injection significantly increased the mechanical thresholds of the ipsilateral hind paw (Figure 7H). Specific activation of inhibitory neurons by blue light stimulation reversed these analgesic effects. In the OF test, activation of neither projection neurons nor inhibitory neurons in the dmPFC affected the total distance (Figure 7I). At the same time, specific activation of inhibitory neurons worsened the CPNL-induced reduction in the percentage of time spent in the central area (Figure 7J). However, in the EPM test, specific activation of inhibitory neurons significantly reversed the activation of projection neuron-induced antianxiety effects (Figure 7, K and L). These results provide functional evidence that local inhibitory neurons in the dmPFC innervated the projection neurons on the $\mathrm{dmPFC} / \mathrm{vlPAG}$ pathway and that the activation of inhibitory neurons in the dmPFC induced anxiety-like behaviors and even worsened chronic pain-induced anxiety-like behaviors through the $\mathrm{dmPFC} / \mathrm{vlPAG}$ descending pathway. 
A

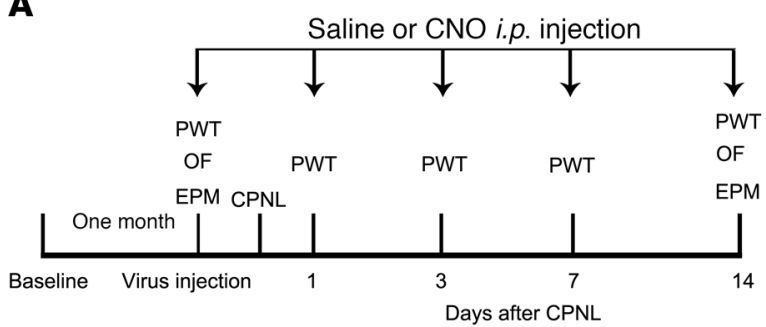

B
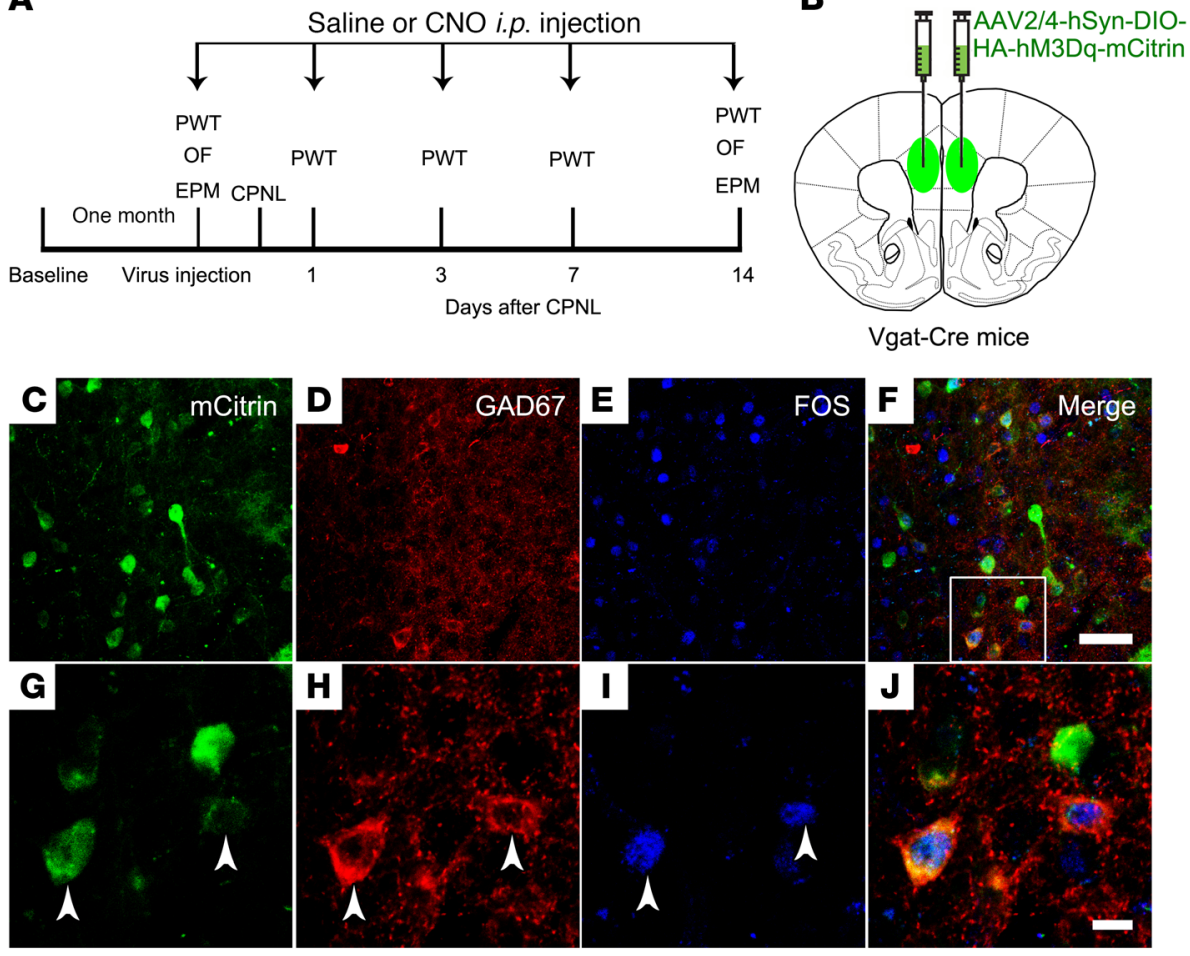

$\mathbf{K}$ * mCitrin - before CNO $\theta \mathrm{hM} 3 \mathrm{Dq}$ - before CNO $¥$ mCitrin - after CNO $\diamond \mathrm{hM} 3 \mathrm{Dq}-$ after CNO $n=8$ per group

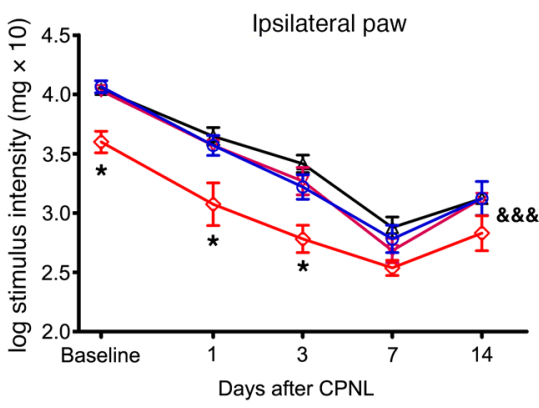

$\mathbf{L}$

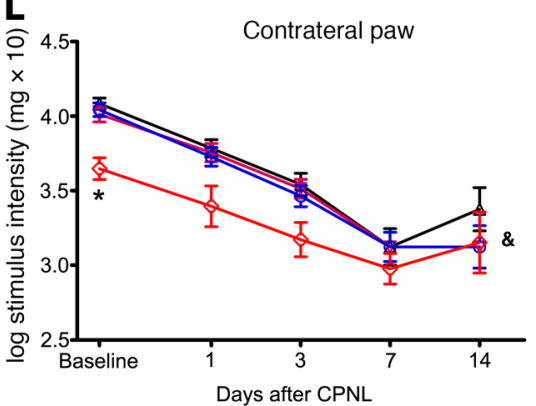

Open field test

Elevated plus maze

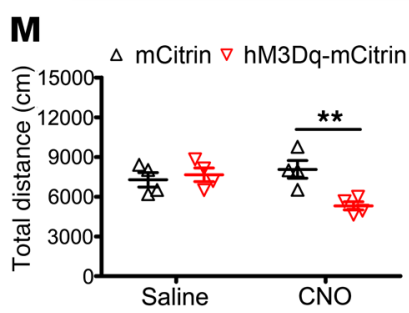

\section{$\mathbf{N}$}

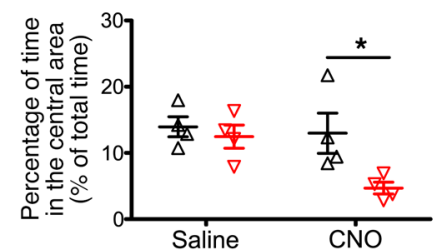

Q

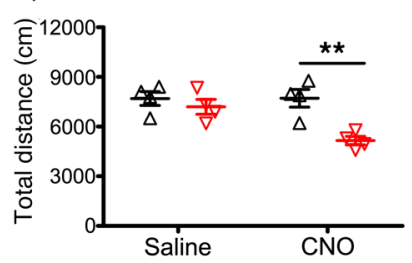

$\mathbf{R}$

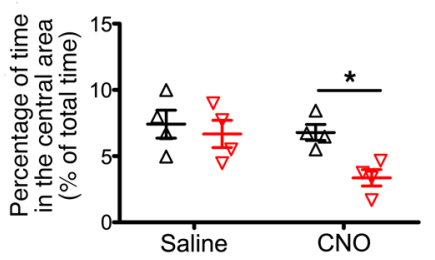

0

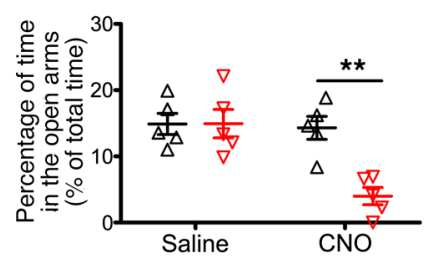

S

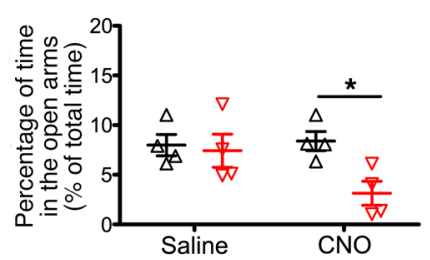

$\mathbf{P}$

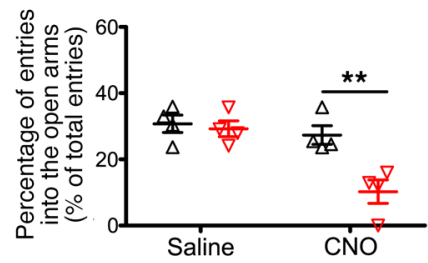

T

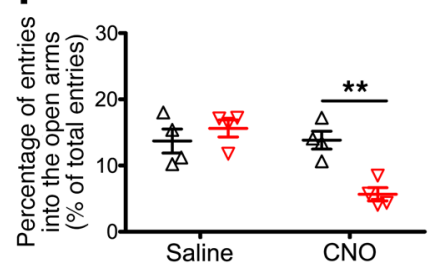

Figure 6. Chemogenetic activation of inhibitory neurons in the dmPFC worsens CPNL-induced mechanical hyperalgesia and anxiety-like behaviors. (A and B) Representative schematic diagrams of hM3Dq virus injection into the dmPFC of Vgat-Cre mice and the procedures for the behavioral test. (C-J) Triple staining of mCitrin (green), GAD67 (red), and FOS (blue) in the dmPFC after CNO i.p. injection. White arrowheads indicate the coexpression of mCitrin, GAD67, and F0S. Scale bars: $40 \mu \mathrm{m}$ (C-F); $10 \mu \mathrm{m}$ ( $\mathbf{G}-\mathbf{J})$. (K and $\mathbf{L}$ ) Mechanical thresholds of the bilateral hind paws following injection of control virus or hM3Dq virus and CNO $1 \mathrm{mg} / \mathrm{kg}$ i.p. injection (hM3Dq virus, ipsilateral: $F_{(1,56)}=19.53, P<0.001$; contralateral: $F_{(1,56)}=5.65, P<0.05 ;$ control virus, ipsilateral: $F_{(1,56)}=1.46, P=0.24$; contralateral: $F_{(1,56)}=1.09, P=0.31$; ${ }^{\&} P<0.05$ and $\& \& \& P<0.001 ;{ }^{*} P<0.05$; 2-way, repeated-measures ANOVA with Bonferroni's post hoc test; $n=8$ mice per group). (M-P) Total distance traveled and percentage of time spent in the central area in the OF test and percentage of time in and percentage of entries into the OAs in the EPM test with CNO injection under normal conditions $\left(\mathrm{M}: F_{(1,12)}=5.13, P<0.05, t=3.71 ; \mathrm{N}: F_{(1,12)}=6.22, P<\right.$ $0.05, t=2.99 ; \mathbf{0}: F_{(1,12)}=8.85, P<0.01, t=4.22 ; \mathbf{P}: F_{(1,12)}=10.55, P<0.01, t=4.22 ;{ }^{*} P<0.05$ and ${ }^{*} P<0.01 ; 2$-way, repeated-measures ANOVA with Bonferroni's post hoc test; $n=4$ mice per group). (Q-T) Total distance traveled and percentage of time spent in the central area in the OF test and percentage of time spent in and percentage of entries into the OAs in the EPM test with CNO injection, 14 days after CPNL $\left(\mathbf{Q}: F_{(1,12)}=12.87, P<0.01, t=4.23 ; \mathbf{R}: F_{(1,12)}=\right.$ 5.92, $P<0.05, t=2.83 ; \mathbf{S}: F_{(1,12)}=5.32, P<0.05, t=2.95 ; \mathbf{T}: F_{(1,12)}=5.05, P<0.05, t=4.14 ;{ }^{*} P<0.05$ and ${ }^{* *} P<0.01$; 2 -way, repeated-measures ANOVA with Bonferroni's post hoc test; $n=4$ mice per group). 
A
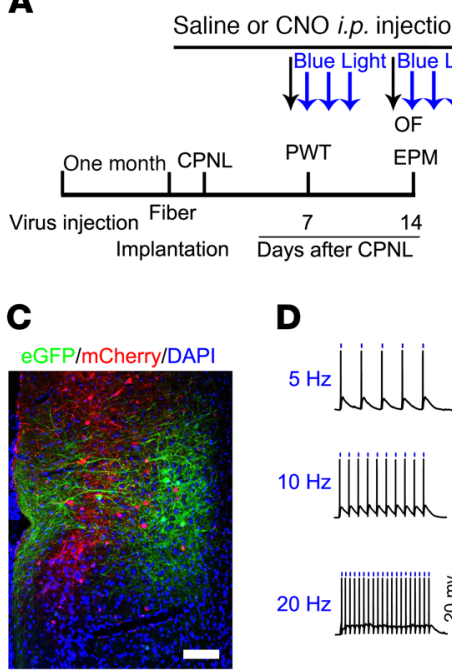

D
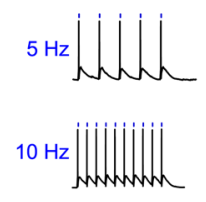

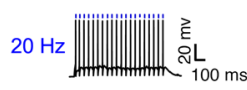

B

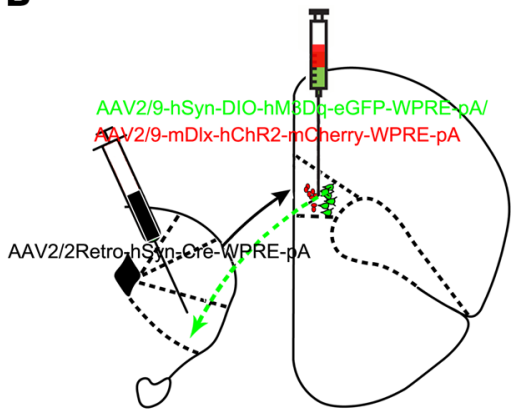

E

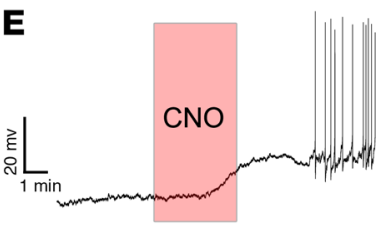

$\mathbf{F}$

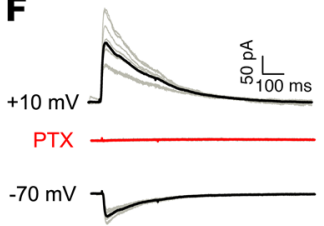

G

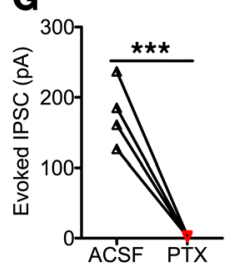

H

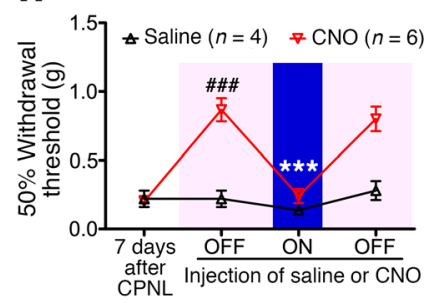

Figure 7. Optogenetic activation of inhibitory neurons in the dmPFC reversed activation of $d m P F C / v I P A C$ pathway-induced analgesic and antianxiety behaviors. (A and B) Representative schematic diagrams of hChR2 and hM3Dq viruses injected into the dmPFC of $C 57$ mice, retro-Cre virus injected into the vIPAG of [57BL/6] mice, and the procedures for the behavioral tests. (C) The triple staining of EGFP (green), mCherry (red), and DAPI (blue) in the dmPFC. Scale bar: $100 \mu \mathrm{m}$. (D) Representative recordings of action potential firing of inhibitory neurons in response to $5 \mathrm{~Hz}, 10 \mathrm{~Hz}$, and $20 \mathrm{~Hz}$ light photostimulation. (E) Whole-cell current-clamp recording from hM3Dqexpressing dmPFC-vIPAC projection neurons. A brief bath application of $40 \mu \mathrm{M}$ CNO (red box) caused a depolarization and action potential firing. (F and $\mathbf{G}$ ) Sample traces showing that light-evoked IPSCs in dmPFC-vIPAC projection neurons were blocked by bath application of PTX ( ${ }^{* * *} P<$ 0.01 , Student's $t$ test). (H) Mechanical thresholds of injured hind paws with CNO injection and blue light stimulation 7 days after CPNL (CNO, $F_{(3,15)}=27.14, P<0.001$, ${ }^{* *} P<0.01$; saline: $F_{(3,9)}=1.03, P=0.426 ; 1$-way, repeated-measures ANOVA with Tukey's post hoc test; ${ }^{\# \#} P<0.001$, Student's $t$ test). (I and J) Total distance traveled and percentage of time in the central area in the OF with CNO injection and blue light stimulation 14 days after CPNL (I: CNO, $F_{(3,21)}=$ 2.64, $P>0.05$; saline: $F_{(3,9)}=1.03, P=0.426$; J: $\mathrm{CNO}, F_{(3,21)}=$ 5.25, $P<0.01$, ${ }^{*} P<0.05$; saline: $F_{(3.9)}=11.47, P<0.01$, ${ }_{\$ \$} P<0.01$; 1-way, repeated-measures ANOVA with Tukey's post hoc test). ( $\mathbf{K}$ and $\mathbf{L}$ ) The percentage of time in and percentage of entries into the OAs in the EPM with CNO injection and blue light stimulation at 14 days after CPNL (K: CNO, $F_{(3,15)}=55.60, P<0.001$, ${ }^{* *} P<0.01$; saline: $F_{(3,9)}=$ 4.86, $P=0.028,{ }^{\$} P<0.05$; L: CNO, $F_{(3,15)}=83.05, P<0.001$, ${ }^{* * *} P<0.01$; saline: $F_{(3,9)}=6.38, P=0.013,{ }^{\$} P<0.05 ; 1$-way, repeated-measures ANOVA with Tukey's post hoc test; $\# \# P<0.001$, Student's $t$ test).

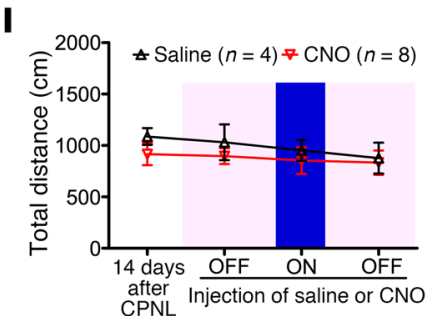

K

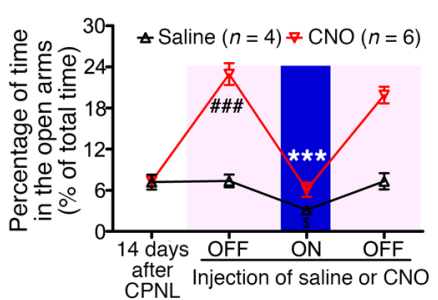

J

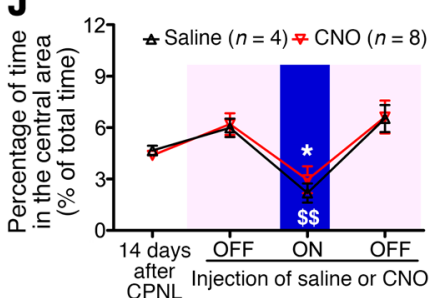

$\mathbf{L}^{\text {(⿻) }}$

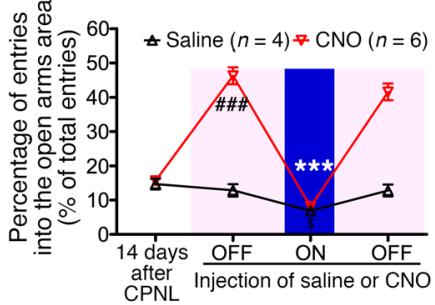

Both the $G A B A_{A} R$ and $m G l u R 1$ in the $d m P F C$ are involved in chronic pain sensations and anxiety-like behaviors. The above results showed that lesions of the dmPFC or deactivation of the dmPFC/ vlPAG neural pathway exacerbated mechanical hyperalgesia and anxiety-like behaviors in the CPNL model. However, the potential molecular mechanism remain unknown. A previous study has shown that the $\mathrm{GABA}_{\mathrm{A}} \mathrm{R}$ and $\mathrm{mGluR} 1$ in the $\mathrm{dmPFC}$ are involved in deactivation of the $\mathrm{dmPFC}$ in an arthritis pain model (19). We performed Western blotting and observed a significant increase in $\mathrm{GABA}_{\mathrm{A}} \mathrm{R}$ and mGluR1 expression in the $\mathrm{dmPFC} 7$ days after CPNL (Supplemental Figure 7). The mechanical thresholds and behaviors in the OF and EPM tests under normal and chronic pain con- ditions were assessed using behavioral pharmacologic approaches (Figure 8, A and B). Many FOS-expressing neurons and cannula placement in the dmPFC indicated that the specific antagonists of the $\mathrm{GABA}_{\mathrm{A}} \mathrm{R}$ and mGluR1 were reliably injected into the dmPFC and functionally activated some neurons (Figure 8, C-E).

Under normal conditions, the mechanical thresholds of the bilateral hind paws were not changed by injection of bicuculline $(0.1 \mu \mathrm{g})$ or LY367385 (specific mGluR1 antagonist, $0.4 \mathrm{nmol}$ ) into the dmPFC (Figure 8, F and G). However, bicuculline or LY367385 injections into the dmPFC produced obvious analgesic effects 7 days after CPNL. There were no obvious differences in the mechanical thresholds between the bicuculline-treated group 
A

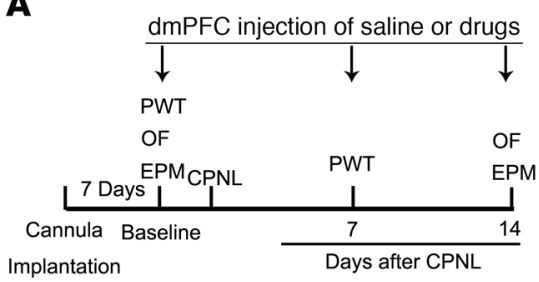

B
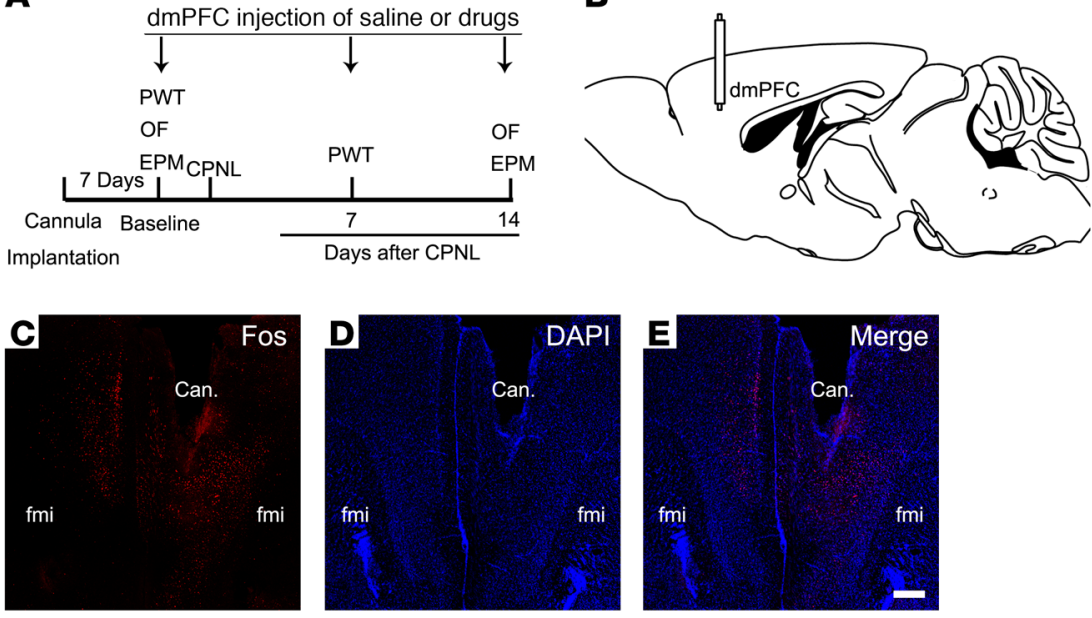

$\mathbf{F}$

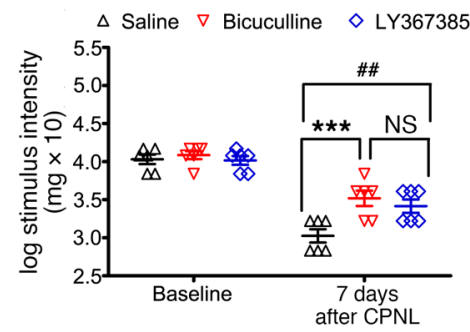

H
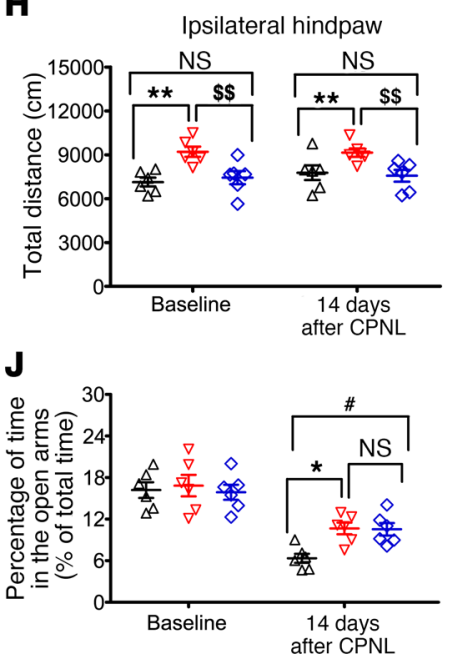

G

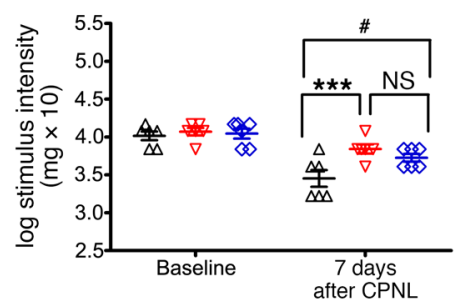

I
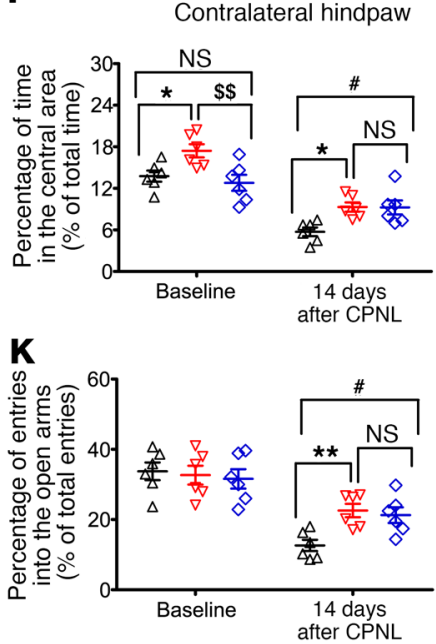

Figure 8. Suppression of GABA $R$ and $m G$ luR 1 in the dmPFC reverses CPNL-induced mechanical hyperalgesia and anxiety-like behaviors. ( $A$ and $B$ ) Representative schematic diagram of cannula placement in the dmPFC and the behavioral testing procedure. (C-E) Double staining of FOS-ir neurons (red) and DAPI (blue) in the dmPFC after drug infusion. Scale bar: $200 \mu \mathrm{m}$ (E) (applied in C and D) Can., cannula; fmi, forceps minor of the corpus callosum ( $\mathbf{F}$ and $\mathbf{G}$ ) Mechanical thresholds of the bilateral hind paws following dmPFC injections of saline, bicuculline 0.1 $\mu \mathrm{g}$ or LY $3673850.4 \mathrm{nmol}$ at baseline and 7 days after CPNL (ipsilateral, $F_{(2,15)}=6.87, P<0.001$ : baseline $t=$ $0.51, t=0.14$; CPNL $t=4.55, t=4.55$; contralateral, $F_{(2,15)}=7.56, P<0.001$ : baseline $t=0.56, t=0.31$; CPNL $t=3.98, t=3.60 ;{ }^{\#} P<0.05$ and ${ }^{\# \#} P<0.01 ;{ }^{* * *} P$ $<0.001$; 2-way, repeated-measures ANOVA with Bonferroni's post hoc test; $n=6$ mice per group). ( $\mathbf{H}$ and $\mathbf{I}$ ) The total distance traveled and percentage of time in the central area in the OF following dmPFC injection of saline, bicuculline or LY 367385 at baseline and 14 days after CPNL (total distance, $F_{(2,15)}=10.60, P$ $<0.01$ : baseline $t=3.79, t=0.55$; CPNL $t=2.85, t=$ 0.38 ; time percentage in the central area, $F_{(2,15)}=6.56$, $P<0.01$ : baseline $t=2.91, t=0.77$; CPNL $t=2.52, t=$ 2.80; ${ }^{*} P<0.05$; ${ }^{*} P<0.05$ and ${ }^{* *} P<0.01$; ${ }^{\$ \$} P<0.01$; 2-way, repeated-measures ANOVA with Bonferroni's post hoc test; $n=6$ mice per group). (J and $\mathbf{K}$ ) The percentage of time in and percentage of entries into the OAs in the EPM following dmPFC injection of saline, bicuculline, or LY 367385 at baseline and 14 days after CPNL (time percentage in the OAs, $F_{(2,15)}$ $=2.96, P>0.05$ : baseline $t=0.43, t=0.21$; CPNL $t$ $=2.86, t=2.78$; entries percentage in the $\mathrm{OAs}, F_{(2,15)}$ $=2.35, P>0.05$ : baseline $t=0.33, t=0.66$; CPNL $t$ $=3.04, t=2.64 ;{ }^{\#} P<0.05 ;{ }^{*} P<0.05$ and ${ }^{* *} P<0.01$; 2-way, repeated-measures ANOVA with Bonferroni's post hoc test; $n=6$ mice per group). and the LY367385-treated group. Under normal conditions, bicuculline injection significantly increased the total distance traveled and the percentage of time spent in the central area in the OF test (Figure 8, H and I). However, LY367385 injection had no obvious influence on those behaviors. Neither bicuculline nor LY367385 $\mathrm{dmPFC}$ injections affected the percentage of time spent in or the percentage of entries into the OAs in the EPM test (Figure 8, J and $\mathrm{K})$. Fourteen days after CPNL, the total distance traveled and the percentage of time spent in the central area in the OF test were significantly increased following bicuculline injection into the dmPFC (Figure 8, H and I). However, only LY367385 injection increased the percentage of time spent in the central area. In the EPM test, both the percentage of time in and percentage of entries into the OAs were significantly increased following bicuculline or LY367385 injections (Figure 8, J and K). The above results indicate that inhibition of $\mathrm{GABA}_{\mathrm{A}}$ Rs or mGluR1s might be a useful approach to rescue hyperalgesia and anxiety-like behaviors under chronic pain conditions.

\section{Discussion}

In this study, we identified an excitatory "top-down" neural pathway from the dmPFC to the vIPAG that used glutamate as a neurotransmitter and acted on VGLUT2-containing neurons in the vlPAG. Under normal conditions, the dmPFC/vlPAG neural pathway played important roles in maintaining the mechanical thresholds of the hind paws and normal behavior in the OF and 
EPM tests. Deactivation of the dmPFC/vlPAG neural pathway by chemical lesions in the dmPFC or specific activation of inhibitory neurons in the dmPFC reduced the mechanical thresholds of hind paws under both normal and chronic pain conditions and induced anxiety-like behaviors. Moreover, the activation of inhibitory neurons in the $\mathrm{dmPFC}$ worsened negative emotions under chronic pain conditions. Analgesic and antianxiety effects were observed after activating the dmPFC/vlPAG neural pathway using optogenetic approaches. We used behavioral pharmacologic approaches, which showed the $\mathrm{GABA}_{\mathrm{A}} \mathrm{R}$ and the mGluR1 in the dmPFC to be involved in the initiation and maintenance of chronic pain and negative emotions. Given the results of the present study, we speculated that deactivation of the $\mathrm{dmPFC} /$ vlPAG neural pathway initiated and maintained mechanical hyperalgesia and CPNLinduced anxiety-like behaviors under chronic pain conditions. Thus, these processes might be the underlying mechanism for the chronic pain cycle and might therefore represent a potential target for the treatment of chronic pain in clinical practice.

GABAergic inhibitory interneurons were found to be involved in the modulation of excitatory pyramidal neurons in the dmPFC $(31,32)$. Our present study showed that specific activation of pyramidal neurons in the $\mathrm{dmPFC}$ produced analgesic and antianxiety effects. However, specific activation of inhibitory neurons in the dmPFC worsened CPNL-induced mechanical hyperalgesia and anxiety-like behaviors. Only lesions of the contralateral, not the ipsilateral, PL attenuated complete Freund's adjuvant-induced (CFA-induced) anxiety-like behaviors and heat hyperalgesia (10), which was contradictory to our results showing that bilateral lesions in the dmPFC worsened CPNL-induced mechanical hyperalgesia. Regarding this difference, we cannot exclude the possibility of differential effects of the chemical drugs used in these 2 investigations (quinolinic acid versus KA) on various lesioned neuron types. Different chronic pain models (CFA versus CPNL) and different areas damaged by the chemical lesions also contributed to the different behavioral readout.

The presence of tactile allodynia in persistent neuropathic pain may be associated with a shift in brain responses toward neural circuits that regulate the affective and motivational components of pain (33). Some clinical studies showed that working memory and attention impairments accompany chronic pain in patients $(34,35)$. These findings indicate that there is a special area in the cerebral cortex involved in the modulation of pain, emotion, and cognition. One study showed that activation of the anterior cingulate cortex (ACC) is involved in chronic pain accompanied by anxiety and/or depression (36). Other studies showed that ACC neurons in layer V were found to project directly to the superficial laminae of the spinal dorsal horn and that stimulation of the ACC facilitates neuropathic pain responses $(37,38)$. Another study showed that the nucleus accumbens (NAc) contributes to an aversive learning signal that leads to sustained pain intensity over time following peripheral nerve injury (21). Deactivation of the NAc plays a causal role in the neuropathic pain phenotype (8). The mPFC-NAc connection is an accurate predictor of the transition from subacute to chronic pain. It has also been reported that the PFC is involved in thalamic-PAG-dorsal horn top-down modulation of pain (39). Activation of PL excitatory neurons strongly activates the descending inhibitory pathway, which thus increases the pain threshold. Huang et al. recently showed the existence and antinociceptive function of the dmPFC/ vlPAG pathway (23). Their study used various tracers and viruses to investigate the projections from the MPFC to the vlPAG. However, the synaptic evidence was not consolidation. In our study, the retrotranssynaptic and anterotranssynaptic viruses were adopted to confirm, for the first time to our knowledge, the specific projections from the dmPFC to the vlPAG.

Specific activation of pyramidal neurons in the PL produced analgesic effects and improved anxiety-like behaviors following CFA injection (10). Furthermore, specific silencing of parvalbumin-containing (PV-containing) neurons in the dmPFC was shown to exert obvious analgesic effects, and specific activation of these neurons worsened chronic pain-induced mechanical and heat hyperalgesia (32). Conditioned place preference scores and place escape/avoidance behavior have also been influenced by these PV-containing neurons in the PL (32). However, the study by Huang et al. showed that optogenetic activation or deactivation of amygdala-PFC inputs did not affect anxiety-like behaviors in a spared nerve injury (SNI) mouse model (23). Their study mainly focused on the analgesic effects of the dmPFC/ vlPAG neural pathway. However, we observed the effects of different neurons in the $\mathrm{dmPFC}$ and the $\mathrm{dmPFC} / \mathrm{vlPAG}$ neural pathway on the nociception-related affective behaviors. Our results indicated that specific activation of the $\mathrm{dmPFC} / \mathrm{vlPAG}$ neural pathway produced both analgesic and antianxiety effects in the CPNL chronic pain mouse model.

In clinical practice, anodal transcranial current stimulation over the left dorsolateral PFC (dlPFC) has been shown to ameliorate neuropathic pain. Analgesia might have occurred through modulation of the emotional pain network (40). In our study, chemogenetic activation of pyramidal neurons in the PFC also produced strong antinociceptive and antianxiety effects. Our results suggested that the $\mathrm{dmPFC}$ was silenced under chronic pain conditions. Specific activation of the dmPFC/vlPAG neural pathway improved anxiety-like behaviors in the EPM test, but not in the OF test, which indicated that the antianxiety effects were not the result of analgesia. Specific activation of VGLUT2-containing neurons or suppression of glutamate decarboxylases-containing (GAD-containing) neurons in the vlPAG induce obvious analgesia and defensive behaviors in the mice $(27,28)$. These various behavioral outcomes suggest that deactivation of the dmPFC/vlPAG neural pathway was involved in the initiation of the anxiety-like behaviors observed in the EPM test.

The vlPAG is a significant modulator of both pain-related and fear behaviors in humans and rodents. Previous studies have shown that specific activation of VGLUT2-containing neurons in the vlPAG produce obvious analgesia and freezing behaviors $(28,41)$. Tovote et al. reported that this freezing behavior was produced by activation or disinhibition of vlPAG excitatory outputs to pre-motor targets in the magnocellular nucleus $(\mathrm{Mc})$ of the medulla (28). However, it is still observed that VGLUT2 ${ }^{+}$neurons could also project to the RVM directly, which is involved in the descending inhibition of pain. Therefore, it was speculated that analgesic and fear behaviors produced by activation of VGLUT2 ${ }^{+}$neurons in the vlPAG depend on the vlPAG/RVM or vlPAG/Mc neuropathway, respectively. Rozeske et al. reported that specific activation 
of the dmPFC-1/vlPAG pathway leads to low-fear states (reducing freezing levels) during contextual fear discrimination, which is similar to our results (42). A likely possibility is that excitatory $\mathrm{dmPFC}$ afferents impinge on the GAD2 ${ }^{+}$neurons in the lateral/ vlPAG (l/vlPAG) (23) and thus inhibit the vlPAG/Mc neuropathway. Besides, inhibition of GAD2 ${ }^{+}$neurons through central amygdala $(\mathrm{CeA})$ inhibitory inputs during cued fear expression would lead to fear responses, as already documented (28). Therefore, the activation of $\mathrm{dmPFC}$ excitatory inputs on the GAD $2^{+}$neurons in the 1/vlPAG would induce the opposite effect, that is, a low-fear state. However, it has still been observed that dmPFC excitatory inputs could also directly project onto VGLUT2 ${ }^{+}$neurons in the vlPAG, which is involved in analgesic behaviors. In fact, the $\mathrm{dmPFC} / \mathrm{vlPAG}$ neuropathway is not the cardinal pathway for modulating anxiety levels. Rozeske et al. reported that modulation of the $\mathrm{dmPFC} / \mathrm{vlPAG}$ pathway in anxiety behaviors is specific to a contextual discrimination paradigm (42). They induced optogenetic activation or inhibition of the $\mathrm{dmPFC} / \mathrm{l} / \mathrm{vlPAG}$ pathway in a classical auditory fear-conditioning and extinction paradigm associated with high- and low-freezing levels. Following conditioning, optogenetic activation of the $\mathrm{dmPFC} / \mathrm{l} / \mathrm{vlPAG}$ pathway did not reduce freezing levels. Huang et al. recently showed that the PFC/vlPAG pathway is critical for the development of mechanical and thermal hypersensitivity after peripheral nerve injury (23). Specific activation of the dmPFC/vlPAG pathway produced both antinociception and antianxiety effects. Nevertheless, we think that the role of the dmPFC/vlPAG in exerting antianxiety effects is not comprehensive. In our study, activation of dmPFC/vlPAG pathway only affected anxiety behaviors in the EPM test but not in the OF test. We hypothesized that the primary function of the dmPFC in anxiety is completed through other targets, such as the amygdala, and that the dmPFC/amygdala pathway is therefore the cardinal pathway for modulation of anxiety levels. Our study also demonstrated that manipulation of the $\mathrm{dmPFC}$ has much stronger effects on anxiety behaviors than does manipulation of the dmPFC/vlPAG pathway.

There were many findings regarding $\mathrm{dmPFC}$ deactivation in different pain models. Several possible molecular targets have been revealed (10). The pain- and anxiety-like behaviors following CFA injection were related to the increased expression of cyclin-dependent kinase 5 (Cdk5). Knockdown of Cdk5 in the PL produced analgesic and antianxiety effects (10). Bilateral intra-mPFC administration of the $\kappa$ opioid receptor (KOR) antagonist norbinaltorphimine (nor-BNI) increased the time spent in the central area in the OF test, suggesting antianxiety effects (43). Behaviorally, acute hyperpolarization-activated cyclic nucleotidegated (HCN-gated) channels blocked by local injection of ZD7288 in the dmPFC of SNI rats induce a decrease in cold allodynia (44). The loss of excitatory cholinergic modulation may also play a critical role in medial PFC ( $\mathrm{mPFC}$ ) deactivation in neuropathic pain and underlie the $\mathrm{MPFC}$-specific cognitive deficits that are comorbid with neuropathic pain (45). Impaired glutamatergic signaling contributes to the general deactivation of layer V PFC neurons following SNI surgery (17). Pain-associated dmPFC suppression has been thought to be driven by hyperactivity of the amygdala inputs to the dmPFC, which results in a group I metabotropic glutamate receptor-mediated increase in local GABAergic activity (19,
20). L-655,708, a selective negative modulator at $\mathrm{GABA}_{\mathrm{A}}$ receptors, was shown to reverse the alterations in hedonic behavior in sucrose preference and social interaction tests (46). Recently, a study by Huang et al. showed that SNI leads to weakened endocannabinoid signaling in layer $\mathrm{V}$ of the $\mathrm{mPFC}$, in turn causing a disinhibition of glutamatergic inputs into inhibitory neurons (23).

The present study is the first to our knowledge to show that the $\mathrm{dmPFC} / \mathrm{vlPAG}$ neural pathway is involved in both pain modulation and anxiety-like behaviors. Activation of this neural pathway might be a straightforward and useful therapeutic strategy to both alleviate chronic pain and rescue anxiety/depression behaviors in chronic pain conditions.

\section{Methods}

Experimental animals. VGLUT2-ires-Cre mice were used to study the inputs of VGLUT2-ir neurons in the vlPAG using a rabies viruslabeling system. Vgat-ires-Cre mice were used to study the effects of local inhibitory neurons in the dmPFC. For the other experiments, C57BL/6J mice were used. All mice (male, 8-10 weeks of age, 20-25 g) were housed under a 12-hour light/12-hour dark cycle, with lights on at 7 am and food and water provided ad libitum. All mice were purchased from The Jackson Laboratory. Mice were housed in clear plastic cages in a controlled environment at a constant temperature of $23^{\circ} \mathrm{C}$ and humidity of $50 \% \pm 10 \%$.

CPNL model. The CPNL model was established to observe painand anxiety-related behaviors following previously described procedures $(37,47)$. Briefly, the mice were anesthetized by i.p. injection of $2 \%$ sodium pentobarbital $(40 \mathrm{mg} / \mathrm{kg}$ ). The CPN was visible between the anterior and posterior groups of muscles, running almost transversely. The left CPN was slowly ligated with chromic gut suture 5-0 until contraction of the dorsiflexor of the foot was visible as twitching of the digits. The skin was then sutured and cleaned. Sham surgery was conducted in the same manner, but the nerve was not ligated. The mice were used for behavioral studies on postsurgical days 1, 3, 7, and 14 .

Measurement of sensitivity to mechanical stimuli. The measurements were based on our previous publications $(48,49)$. Mice were habituated to the testing environment for 3 days before baseline testing and were then placed under inverted plastic boxes $(7 \times 7 \times 10$ $\mathrm{cm}$ ) on an elevated mesh floor and allowed to habituate for 30 minutes before threshold testing. A logarithmic series of 8 calibrated Semmes-Weinstein monofilaments (von Frey hairs, Stoelting) was applied to the plantar surface of the paw that received nerve ligation and to the contralateral paw. Positive responses included licking, biting, and sudden withdrawal of the hind paws. The log stiffness of the hairs was determined by $\log _{10}$ (milligrams $\times 10$ ). A von Frey filament was applied 5 times ( 3 seconds for each stimulus) to each testing area. The minimum bending force of the von Frey filament able to evoke 3 occurrences of the paw withdrawal reflex was expressed as a 50\% withdrawal threshold. The stimulus was stopped if the threshold exceeded $10.0 \mathrm{~g}$ (cutoff value). Mechanical allodynia was assessed on the basis of the responsiveness of the hind paws to the application of a particular von Frey filament $(0.04 \mathrm{~g})$. Ten trails were carried out each time, and the results are expressed as a percentage of positive responses. All tests were performed in a blinded manner.

OF and EPM tests. The OF test was conducted according to the method used in our previous studies $(50,51)$. Mice were placed at the center of a cubic chamber that measured $50 \mathrm{~cm}$ (width) $\times 50 \mathrm{~cm}$ 
(length) $\times 45 \mathrm{~cm}$ (height). The locomotor activity of mice for 15 minutes was monitored by an automated analysis system. The total distance traveled was used as a measure of the locomotion, and the percentage of time spent in the central area (percentage of total time) was used to evaluate anxiety-like behavior by offline analysis. All animals were habituated to the testing room for 30 minutes before the OF test. The behavioral room was dimly illuminated with indirect white lighting.

The EPM test was conducted according to methods described in our previous studies $(52,53)$. The EPM was made of a white Plexiglass apparatus consisting of 2 opposing OAs $(30 \times 5 \mathrm{~cm}), 2$ opposing closed arms (CAs) $(30 \times 5 \times 25 \mathrm{~cm})$, and a central area measuring $5 \times 5 \mathrm{~cm}$. The plus-shaped platform was $50 \mathrm{~cm}$ above the floor. Generally, mice were placed individually into the center area of the maze facing one of the OAs and were allowed to explore for 5 minutes. The number of $\mathrm{OA}$ and CA entries and time spent in the OAs and CAs were recorded by an automated analysis system. Only when all 4 paws crossed out of the central zone was the animal considered in an OA or a CA. The percentage of time spent in the OAs (percentage of total time) and the percentage of entries into the OAs (percentage of total entries) were measured to evaluate general anxiety levels. The environmental conditions and the habituation protocol for the EPM test were the same as those of the OF test.

Stereotaxic KA, tracer, and virus injection. The injection procedures have been described in our previous studies $(54,55)$. In brief, mice were anesthetized with $2 \%$ sodium pentobarbital $(40 \mathrm{mg} / \mathrm{kg}$, i.p.). A midline opening was made in the skull with a dental drill to insert a glass micropipette connected to a microsyringe ( $1 \mu \mathrm{L}$, Hamilton) into the target site. Detailed injection information is presented in Supplemental Table 1 . The mice with the virus infection were allowed to recover for 4 weeks before the behavioral experiments (56).

For cell-type-specific retrograde transsynaptic tracing, Credependent AAV2/9-DIO-EGFP-TVA and AAV2/9-DIO-RG were mixed at equal volumes before virus injections. Then, $300 \mathrm{~nL}$ of the AAV mixture was stereotaxically injected into the vlPAG of VGLUT2-ires-Cre mice, which allowed EGFP-TVA- and RGselective expression in VGLUT2-containing neurons. After 4 weeks of recovery and AAV expression, $300 \mathrm{~nL}$ RV-EnvA- $\Delta \mathrm{G}$-dsRed was injected into the same location in a biosafety level-2 environment. After 1 week of rabies virus infection and transsynaptic spread (57, 58), the animals were sacrificed.

Fiber optic ferrules or cannula implantation. For optogenetic experiments, a custom-made ferrule with a fiber optic cable $(100 \mu \mathrm{m}$ in core diameter, Doric Lenses) was subsequently placed above the vlPAG and fixed on the skull with bone screws, super glue, and dental cement (Metabond, Parkell). Unless otherwise indicated, the control animals used in this study were animals with the same genetic background that were injected with AAV2/2-CaMKII $\alpha$-EYFP and that also received fiber optic implantation into the vlPAG.

For microinjection of drugs into the $\mathrm{dmPFC}$, a $3.0 \mathrm{~mm}$ long guide cannula (6202, OD $0.56 \times$ ID $0.38 \mathrm{~mm}$, RWD Life Science) was stereotaxically implanted, aimed at the contralateral $\mathrm{dmPFC}$, fixed to the skull with bone screws, super glue, and dental cement, and then a dummy cannula was inserted into the guide cannula. After guide cannula implantation and a 1-week recovery, mice were tested for pain-related and anxiety-like behaviors.

Behavioral tests using optogenetic, chemogenetic, and pharmacological approaches. Optogenetic experiments were performed in a man- ner similar to those described in previous studies with modification $(10,59)$. Mice with fiber optic ferrules in the vlPAG were photostimulated by attaching the ferrule to a fiber optic cable with a rotary joint (Doric Lenses), followed by another fiber optic cable that was then attached to a fiber-coupled $473 \mathrm{~nm}$ blue laser (BOGS-1, Beijing BioGold Biotech Co. Ltd). Laser power output from the fiber optic cable was measured using a photometer (Thorlabs) and set to approximately $12 \mathrm{~mW} / \mathrm{mm}^{2}$, as power loss through the ferrule-cable connection was 10\%-20\%. For the von Frey test, a 2-minute stimulation (473 nM at $20 \mathrm{~Hz}, 10 \mathrm{~ms}$ pulse) with a 2-minute break following each stimulation was performed in each animal until the mechanical thresholds were detected. For the OF and EPM tests, behavior over a total of 9 minutes was recorded and included 3 minutes of photostimulation, a 3-minute break, and 3 minutes of photostimulation with the same blue light frequency and power intensity.

For the chemogenetic test, the mice, following virus injection, received an i.p. injection of $1 \mathrm{mg} / \mathrm{kg}$ CNO (MilliporeSigma) or $50 \mu \mathrm{L}$ saline, followed by detection of the pain thresholds and the recording of behaviors in the OF and EPM.

Saline (0.4 $\mu \mathrm{L})$, bicuculline (0.1 $\mu \mathrm{g}$; MilliporeSigma), or LY367385 (0.4 nmol; Tocris Bioscience) was injected at a rate of $50 \mathrm{~nL} / \mathrm{min}$ using a $3.2 \mathrm{~mm}$ long injection cannula connected to a Pump Elite 11 infusion pump (Harvard Apparatus) via a polyethylene tube. Behavioral assessments of mice were performed by observers blinded to the animal groups. Likewise, the mechanical thresholds of the bilateral hind paws and behaviors of the mice in the OF and EPM tests were detected or recorded.

Immunohistochemical and immunofluorescence histochemical staining. The procedures were performed as previously described $(48,55)$. After deep anesthesia using pentobarbital $(100 \mathrm{mg} / \mathrm{kg}$, i.p.) and perfusion with $0.1 \mathrm{M}$ phosphate buffer (PB) $(\mathrm{pH} 7.4)$ containing $4 \%$ paraformaldehyde, the whole brain was removed and postfixed for 4 hours and then immersed in $30 \%$ sucrose in $0.1 \mathrm{M} \mathrm{PB}$ for 48 hours at $4^{\circ} \mathrm{C}$. Transverse forebrain containing $\mathrm{dmPFC}$, vlPAG, or RVM sections were cut at $40 \mu \mathrm{m}$ thickness on a cryostat (Leica CM1800) at $-20^{\circ} \mathrm{C}$, and the sections were collected serially into dishes containing 0.01 M PBS (pH 7.4).

Immunohistochemical staining with FG or BDA was performed with an avidin-biotin-peroxidase complex (ABC) method. The sections were blocked for 0.5 hours at room temperature (RT) $\left(20^{\circ} \mathrm{C}-25^{\circ} \mathrm{C}\right)$. Next, the sections were incubated overnight at RT with guinea pig (Gp) anti-FG antiserum (1:200; NM-101, Protos Biotech), followed by incubation with donkey anti-Gp IgG (1:500; AP193B, MilliporeSigma) for 5 hours. Finally, sections (or sections used for detection of BDA) were incubated with the ABC Elite Kit (1:100; A-2001, Vector Laboratories) at RT for 2 hours and then were further incubated in a solution containing $0.05 \mathrm{M}$ Tris- $\mathrm{HCl}, 0.13 \%$ diaminobenzidine, and $0.005 \%$ hydrogen peroxide for 20 to 30 minutes to complete the diaminobenzidine reaction. Finally, all sections were mounted onto gelatin-coated glass slides, air dried, dehydrated in a graded series of diluted ethanol, cleared in xylene, and coverslipped.

The procedures for immunofluorescence histochemical staining were the same as those previously described $(55,60,61)$. The dmPFC sections from the mice that received FG or virus injection, or from mice that underwent cannula implantation were used to evaluate the double staining of FG/CaMKII, GAD67/FG, NeuN/FG, NeuN/GFAP, or FOS/ DAPI or biocytin/DAPI, or the triple staining of mCitrin/GAD67/FOS. The vlPAG sections from mice that received the dmPFC AAV2/2-CaMKII $\alpha$-EYFP injection and the RVM FG injection, or from mice that 
received the dmPFC AAV2/2-CaMKII $\alpha$-hChR2-EYFP injection were used to evaluate the double staining of EYFP/FG and EYFP/FOS or the triple staining of EYFP/FG/synapsin (Supplemental Table 2). Finally, all of the sections were then mounted onto glass slides and observed with laser scanning confocal microscopy (FV1000, Olympus) under appropriate filters.

ISH histochemistry combined with retrograde tract tracing. FISH was performed as previously described $(62,63)$. After a survival period of 1 week, the mice injected with FG into the vlPAG or RVM were euthanized and then perfused, and the brains were dissected and cut into $20 \mu \mathrm{m}$ thick transverse sections. Then, the sections were hybridized with digoxigenin-labeled VGLUT1 or VGLUT2 riboprobe. After washes and ribonuclease A treatment, the hybridized sections were incubated overnight at RT with a mixture (1:2000 dilution) of peroxidaseconjugated anti-digoxigenin sheep antibody (11-207-733-910, Roche Diagnostics) and Gp anti-FG antibody (1:200 dilution). To amplify the signals for VGLUT1 or VGLUT2 mRNA, we used the biotinylated tyramine (BT) glucose oxidase (GO) amplification method (64). Subsequently, the sections were incubated with $10 \mu \mathrm{g} / \mathrm{mL}$ Alexa Fluor 488-conjugated streptavidin (S-11223, Invitrogen, Thermo Fisher Scientific) for 2 hours and then with $10 \mu \mathrm{g} / \mathrm{mL}$ Alexa Fluor 594conjugated goat antibody against Gp IgG (Invitrogen) for 4 hours. Finally, all of the sections were mounted onto glass slides and observed with laser scanning confocal microscopy.

Electrophysiology. Coronal brain slices containing dmPFC were prepared according to methods described in our previous studies $(60,65)$. Transverse slices $(300 \mu \mathrm{m}$ thick) were cut on a vibrating microtome (Leica VT 1200s) at $4^{\circ} \mathrm{C}$. The slices were transferred into a submerged chamber containing artificial cerebrospinal fluid containing the following reagents: $124 \mathrm{mM} \mathrm{NaCl}, 2.5 \mathrm{mM} \mathrm{KCl}, 2$ $\mathrm{mM} \mathrm{CaCl}_{2}, 2 \mathrm{mM} \mathrm{MgSO}_{4}, 25 \mathrm{mM} \mathrm{NaHCO}_{3}, 1 \mathrm{mM} \mathrm{NaH}_{2} \mathrm{PO}_{4}, 10 \mathrm{mM}$ glucose, $1 \mathrm{mM}$ ascorbate, and $3 \mathrm{mM}$ sodium pyruvate. The recordings were made in voltage-clamp or current-clamp mode using the Axon 700B amplifier (Axon Instruments). Pclamp software, version 10.02 (Axon Instruments) was used to acquire and analyze the data. The recording pipettes (3-6 $\mathrm{M} \Omega$ ) filled with a solution containing $130 \mathrm{mM}$ potassium-gluconate, $5 \mathrm{mM} \mathrm{NaCl}, 15 \mathrm{mM} \mathrm{KCl}, 0.4$ mM EGTA, 10 mM HEPES, 4 mM Mg-ATP, and $0.3 \mathrm{mM} \mathrm{Na}_{2}$-GTP (adjusted to pH 7.3 with $\mathrm{KOH}$ ) were used for recording. For spontaneous excitatory postsynaptic current (sEPSC) recordings, cells were voltage clamped at $-70 \mathrm{mV}$. The recordings were performed on retroBead-containing pyramidal neurons, EGFP-expressing pyramidal neurons, or mCherry-expressing inhibitory neurons in the $\mathrm{dmPFC}$ that were visualized under epifluorescence using a filter set (U-HGLGPS, Olympus) with a monochrome CCD camera (IR-1000E, DAGE-MTI) and monitor. In cases of retroBeads tracing, $0.2 \%$ biocytin (MilliporeSigma) was introduced into the recording solution to identify the recorded neurons. The action potentials were detected in response to suprathreshold current injections in the current-clamp mode. Depolarizing currents of 0-300 pA (400 $\mathrm{ms}$ duration) were delivered in increments of $20 \mathrm{pA}$. Optical stimulation $(473 \mathrm{~nm})$ was applied with a custom laser fiber. The stimulation pattern was similar to that used in vivo $(5 \mathrm{~Hz}, 10 \mathrm{~Hz}$, and $20 \mathrm{~Hz}$ pulse trains). Blue light was applied through a 40 objective over the field of view of the patched cell. Picrotoxin $(100 \mu \mathrm{M})$ was used to block inhibitory synaptic transmission from the inhibitory neurons to the $\mathrm{dmPFC} / \mathrm{vlPAG}$ projection neurons. CNO-evoked currents were recorded in voltage-clamp mode with the membrane potential held at $70 \mathrm{mV}$, and cells were stimulated using $40 \mu \mathrm{M}$ CNO.

Western blot analysis. Mice were sacrificed after inducing deep anesthesia using pentobarbital $(100 \mathrm{mg} / \mathrm{kg}$, i.p.), and then the whole brain was quickly removed. The dmPFC was collected and homogenized with a hand-held pestle in SDS sample buffer. The electrophoresis samples were heated at $100^{\circ} \mathrm{C}$ for 5 minutes and loaded onto $10 \%$ SDS-polyacrylamide gels with standard Laemmli solutions (Bio-Rad Laboratories). The proteins were electroblotted onto a PVDF membrane (Immobilon-P, MilliporeSigma). The membranes were placed in a blocking solution for 1 hour and incubated overnight under gentle agitation with the primary antibody rabbit anti-GABA $\mathrm{R}$ (1:1000; ab72446, Abcam) or rabbit anti-mGluR1 (1:1000; ab82211, Abcam) and mouse anti- $\beta$ actin (1:5000; MilliporeSigma). Bound primary antibodies were detected with an HRP-conjugated anti-rabbit antibody (1:5000; ZB-2301, ZSGB-Bio) or anti-mouse secondary antibody (1:5000; ZB-2305, ZSGB-Bio). All reactions were detected by the ECL detection method. The densities of protein blots were analyzed using Labworks Software (Ultra-Violet Products). The densities of the $\mathrm{GABA}_{\mathrm{A}} \mathrm{R}, \mathrm{mGluR} 1$, and $\beta$-actin immunoreactive bands were quantified with background subtraction.

Statistics. Values are reported as the mean \pm SEM. The behavioral data were analyzed by 1-way or 2-way repeated-measures ANOVA, with Bonferroni's or Tukey's post hoc test, paired $t$ test, or 2-tailed Student's $t$ test. Student's $t$ test was used for immunofluorescence analysis and analysis of Western blot data. The comparison of electrophysiology data was made using a 2-way repeated-measures ANOVA with Bonferroni's post hoc test or Student's $t$ test. Statistical analyses were performed using GraphPad Prism, version 6.0e (GraphPad Software). Normality and equal variance tests were performed for all statistical analyses. A $P$ value of 0.05 or less was considered statistically significant.

Study approval. All experimental procedures received prior approval from the Animal Use and Care Committee for Research and Education of The Fourth Military Medical University (Xi'an, China) and followed ethical guidelines for the study of experimental pain in conscious animals.

\section{Author contributions}

YQL, YLD, XL, and JBY conceived the project and designed the experiments. JBY, FL, ZYW, JH, RR, YNP, HXL, and HL performed immunofluorescence staining. YCL, JBY, TZ, HXL, JY, and TD completed the dmPFC lesion and behavior tests. SHL, Yi Sun, Yan Sun, and TC carried out the optogenetic and chemogenetic investigations. SHL, Yi Sun, and YB performed the pharmacological tests. YQL, YLD, XL, ZFC, and JBY wrote the manuscript. WJZ, JC, YCL, and WDZ completed the supplementary experiments. All authors approved the manuscript.

\section{Acknowledgments}

This work was supported by the National Natural Science Foundation of China (grant 81620108008, to YQL; 31871061 and 31671087, to YLD; 81801099, to JBY); Hainan ZDYF (2018153, to YQL); the China Postdoctoral Science Foundation Special Funded Project (2019TQ0135); and an intramural grant from the Fourth Military Medical University (2015D06, to JBY). JBY was also supported by the China Scholar Council. 
Address correspondence to: Yun-Qing Li or Yu-Lin Dong, Department of Anatomy, Histology and Embryology and K. K. Leung Brain Research Centre, The Fourth Military Medical University, No. 169 Changle West Road, Xi'an 710032, China. Phone: 86.29.84772706; Email: deptanat@fmmu.edu. cn (YQ Li); donganat@fmmu.edu.cn (YL Dong). Or to: Xiang Li, Department of Orthopaedics, The First Affiliated Hospital with Nanjing Medical University, No. 300 Guangzhou Road, 210029, China. Phone: 86.25.68303194; Email: lixiangjsph@163.com.
1. Malfliet A, et al. Brain changes associated with cognitive and emotional factors in chronic pain: A systematic review. Eur J Pain. 2017;21(5):769-786.

2. Simons LE, Elman I, Borsook D. Psychological processing in chronic pain: a neural systems approach. Neurosci Biobehav Rev. 2014;39:61-78.

3. Block CK, Brock J. The relationship of pain catastrophizing to heightened feelings of distress. Pain Manag Nurs. 2008;9(2):73-80.

4. Price DD. Psychological and neural mechanisms of the affective dimension of pain. Science. 2000;288(5472):1769-1772.

5. Gambassi G. Pain and depression: the egg and the chicken story revisited. Arch Gerontol Geriatr. 2009;49 Suppl 1:103-112.

6. Jann MW, Slade JH. Antidepressant agents for the treatment of chronic pain and depression. Pharmacotherapy. 2007;27(11):1571-1587.

7. Jurik A, et al. Roles of prefrontal cortex and paraventricular thalamus in affective and mechanical components of visceral nociception. Pain. 2015;156(12):2479-2491.

8. Lee M, et al. Activation of corticostriatal circuitry relieves chronic neuropathic pain. J Neurosci. 2015;35(13):5247-5259.

9. Martinez E, Lin HH, Zhou H, Dale J, Liu K, Wang J. Corticostriatal Regulation of Acute Pain. Front Cell Neurosci. 2017;11:146.

10. Wang GQ, et al. Deactivation of excitatory neurons in the prelimbic cortex via Cdk5 promotes pain sensation and anxiety. Nat Commun. 2015;6:7660.

11. Barbas H. Anatomic basis of cognitive-emotional interactions in the primate prefrontal cortex. Neurosci Biobehav Rev. 1995;19(3):499-510.

12. Lee D, Rushworth MF, Walton ME, Watanabe M, Sakagami M. Functional specialization of the primate frontal cortex during decision making. J Neurosci. 2007;27(31):8170-8173.

13. Rushworth MF, Noonan MP, Boorman ED, Walton ME, Behrens TE. Frontal cortex and reward-guided learning and decision-making. Neuron. 2011;70(6):1054-1069.

14. Friedman A, et al. A corticostriatal path targeting striosomes controls decision-making under conflict. Cell. 2015;161(6):1320-1333.

15. Salzman CD, Fusi S. Emotion, cognition, and mental state representation in amygdala and prefrontal cortex. Annu Rev Neurosci. 2010;33:173-202.

16. Sun H, Neugebauer V. mGluR1, but not mGluR5, activates feed-forward inhibition in the medial prefrontal cortex to impair decision making. J Neurophysiol. 2011;106(2):960-973.

17. Kelly CJ, Huang M, Meltzer H, Martina M. Reduced glutamatergic currents and dendritic branching of layer 5 pyramidal cells contribute to medial prefrontal cortex deactivation in a Rat Model of Neuropathic Pain. Front Cell Neurosci. 2016;10:133.

18. Metz AE, Yau HJ, Centeno MV, Apkarian AV,
Martina M. Morphological and functional reorganization of rat medial prefrontal cortex in neuropathic pain. Proc Natl Acad Sci USA. 2009;106(7):2423-2428.

19. Ji G, Neugebauer V. Pain-related deactivation of medial prefrontal cortical neurons involves mGluR1 and GABA(A) receptors. J Neurophysiol. 2011;106(5):2642-2652.

20. Ji G, et al. Cognitive impairment in pain through amygdala-driven prefrontal cortical deactivation. J Neurosci. 2010;30(15):5451-5464.

21. Baliki MN, et al. Corticostriatal functional connectivity predicts transition to chronic back pain. Nat Neurosci. 2012;15(8):1117-1119.

22. Mansour AR, et al. Brain white matter structural properties predict transition to chronic pain. Pain. 2013;154(10):2160-2168.

23. Huang J, et al. A neuronal circuit for activating descending modulation of neuropathic pain. Nat Neurosci. 2019;22(10):1659-1668.

24. François A, et al. A brainstem-spinal cord inhibitory circuit for mechanical pain modulation by GABA and enkephalins. Neuron . 2017;93(4):822-839.e6.

25. Millan MJ. Descending control of pain. Prog Neurobiol. 2002;66(6):355-474.

26. Wu SX, Wang W, Li H, Wang YY, Feng YP, Li YQ. The synaptic connectivity that underlies the noxious transmission and modulation within the superficial dorsal horn of the spinal cord. Prog Neurobiol. 2010;91(1):38-54.

27. Samineni VK, et al. Divergent modulation of nociception by glutamatergic and GABAergic neuronal subpopulations in the periaqueductal gray. eNeuro. 2017;4(2):ENEURO.0129-16.2017.

28. Tovote P, et al. Midbrain circuits for defensive behaviour. Nature. 2016;534(7606):206-212.

29. Zingg B, et al. AAV-mediated anterograde transsynaptic tagging: mapping vorticocollicular input-defined neural pathways for defense behaviors. Neuron. 2017;93(1):33-47.

30. Zingg B, Peng B, Huang J, Tao HW, Zhang LI. Synaptic specificity and application of anterograde transsynaptic AAV for probing neural circuitry. J Neurosci. 2020;40(16):3250-3267.

31. Kvitsiani D, Ranade S, Hangya B, Taniguchi H, Huang JZ, Kepecs A. Distinct behavioural and network correlates of two interneuron types in prefrontal cortex. Nature. 2013;498(7454):363-366.

32. Zhang Z, Gadotti VM, Chen L, Souza IA, Stemkowski PL, Zamponi GW. Role of prelimbic GABAergic circuits in sensory and emotional aspects of neuropathic pain. Cell Rep. 2015;12(5):752-759.

33. Chang PC, Centeno MV, Procissi D, Baria A, Apkarian AV. Brain activity for tactile allodynia: a longitudinal awake rat functional magnetic resonance imaging study tracking emergence of neuropathic pain. Pain. 2017;158(3):488-497.

34. Baker KS, Gibson S, Georgiou-Karistianis N, Roth RM, Giummarra MJ. Everyday executive functioning in chronic pain: specific deficits in working memory and emotion control, predicted by mood, medications, and pain interference. Clin J Pain. 2016;32(8):673-680.

35. Moriarty O, McGuire BE, Finn DP. The effect of pain on cognitive function: a review of clinical and preclinical research. Prog Neurobiol. 2011;93(3):385-404.

36. Zhuo M. Neural mechanisms underlying anxiety-chronic pain interactions. Trends Neurosci. 2016;39(3):136-145.

37. Chen T, et al. Postsynaptic potentiation of corticospinal projecting neurons in the anterior cingulate cortex after nerve injury. Mol Pain. 2014;10:33.

38. Chen T, et al. Postsynaptic insertion of AMPA receptor onto cortical pyramidal neurons in the anterior cingulate cortex after peripheral nerve injury. Mol Brain. 2014;7:76.

39. Bingel U, Tracey I, Wiech K. Neuroimaging as a tool to investigate how cognitive factors influence analgesic drug outcomes. Neurosci Lett. 2012;520(2):149-155.

40. Ayache SS, et al. Prefrontal tDCS decreases pain in patients with multiple sclerosis. Front Neurosci. 2016;10:147.

41. Taylor NE, et al. The role of glutamatergic and dopaminergic neurons in the Periaqueductal gray/dorsal raphe: separating analgesia and anxiety. eNeuro. 2019;6(1):ENEURO.0018-18.2019.

42. Rozeske RR, et al. Prefrontal-periaqueductal gray-projecting neurons mediate context fear discrimination. Neuron. 2018;97(4):898-910.e6.

43. Tejeda HA, Hanks AN, Scott L, Mejias-Aponte C, Hughes ZA, O'Donnell P. Prefrontal cortical kappa opioid receptors attenuate responses to amygdala inputs. Neuropsychopharmacology. 2015;40(13):2856-2864.

44. Cordeiro Matos S, Zhang Z, Séguéla P. Periphera neuropathy induces HCN channel dysfunction in pyramidal neurons of the medial prefrontal cortex. J Neurosci. 2015;35(38):13244-13256.

45. Radzicki D, Pollema-Mays SL, Sanz-Clemente A, Martina M. Loss of M1 receptor dependent cholinergic excitation contributes to $\mathrm{MPFC}$ deactivation in neuropathic pain. J Neurosci. 2017;37(9):2292-2304.

46. Fischell J, Van Dyke AM, Kvarta MD, LeGates TA, Thompson SM. Rapid antidepressant action and restoration of excitatory synaptic strength after chronic stress by negative modulators of alpha5-containing GABAA receptors. Neuropsychopharmacology. 2015;40(11):2499-2509.

47. Vadakkan KI, Jia YH, Zhuo M. A behavioral model of neuropathic pain induced by ligation of the common peroneal nerve in mice. JPain. 2005;6(11):747-756.

48. Yin JB, et al. Analgesic effects of Danggui-Shaoyao-San on various "phenotypes" of nociception and inflammation in a formalin pain model. $\mathrm{Mol}$ 
Neurobiol. 2016;53(10):6835-6848.

49. Zhao YQ, et al. The analgesic effects of celecoxib on the formalin-induced short- and long-term inflammatory pain. Pain Physician. 2017;20(4):E575-E584.

50. Wu HH, et al. Inhibiting spinal neuron-astrocytic activation correlates with synergistic analgesia of dexmedetomidine and ropivacaine. PLoS One. 2014;9(3):e92374.

51. Zhai MZ, et al. Dexmedetomidine dosedependently attenuates Ropivacaine-induced seizures and negative emotions via inhibiting phosphorylation of amygdala extracellular signal-regulated kinase in mice. Mol Neurobiol. 2016;53(4):2636-2646.

52. Xia L, Zhai M, Wang L, Miao D, Zhu X, Wang W. FGF2 blocks PTSD symptoms via an astrocyte-based mechanism. Behav Brain Res. 2013;256:472-480.

53. Zhang MM, et al. Effects of NBO01 and gabapentin on irritable bowel syndrome-induced behavioral anxiety and spontaneous pain. $\mathrm{Mol}$ Brain. 2014;7:47.

54. Chen T, et al. Neurokinin-1 receptor-expressing neurons that contain serotonin and gammaaminobutyric acid in the rat rostroventromedial medulla are involved in pain processing. J Pain. 2013;14(8):778-792.

55 . Yin JB, et al. Neurochemical properties of BDNF-containing neurons projecting to rostral ventromedial medulla in the ventrolateral periaqueductal gray. Front Neural Circuits. 2014;8:137.

56. Paxinos G, Watson C, ed. The rat brain in stereotaxic coordinates. Amsterdam: Elsevier; 2005.

57. Wall NR, Wickersham IR, Cetin A, De La Parra M, Callaway EM. Monosynaptic circuit tracing in vivo through Cre-dependent targeting and complementation of modified rabies virus. Proc Natl Acad Sci USA. 2010;107(50):21848-21853.

58. Watabe-Uchida M, Zhu L, Ogawa SK, Vamanrao A, Uchida N. Whole-brain mapping of direct inputs to midbrain dopamine neurons. Neuron. 2012;74(5):858-873.

59. Yu YQ, Barry DM, Hao Y, Liu XT, Chen ZF. Molecular and neural basis of contagious itch behavior in mice. Science. 2017;355(6329):1072-1076.

60. Chen T, et al. Mechanism underlying the anal- gesic effect exerted by endomorphin-1 in the rat ventrolateral periaqueductal gray. Mol Neurobiol. 2016;53(3):2036-2053.

61. Liang SH, et al. Collateral projections from the lateral parabrachial nucleus to the paraventricular thalamic nucleus and the central amygdaloid nucleus in the rat. Neurosci Lett. 2016;629:245-250.

62. Ge SN, et al. Differential expression of VGLUT1 or VGLUT2 in the trigeminothalamic or trigeminocerebellar projection neurons in the rat. Brain Struct Funct. 2014;219(1):211-229.

63. Liu X, et al. Proprioceptive mechanisms in occlusion-stimulated masseter hypercontraction. Eur J Oral Sci. 2017;125(2):127-134.

64. Kuramoto E, Furuta T, Nakamura KC, Unzai T, Hioki H, Kaneko T. Two types of thalamocortical projections from the motor thalamic nuclei of the rat: a single neuron-tracing study using viral vectors. Cereb Cortex. 2009;19(9):2065-2077.

65. Chen T, et al. Top-down descending facilitation of spinal sensory excitatory transmission from the anterior cingulate cortex. Nat Commun. 2018;9(1):1886. 